Observation d'une Venise confinée. Le tourisme, pharmakon vénitien, à la fois remède unique, poison addictif et bouc émissaire

Observation of a Venice in Lockdown. Tourism, Venetian Pharmakon, Both

Unique Remedy, Addictive Poison and Easy Scapegoat

\title{
Prosper Wanner
}

\section{OpenEdition \\ Journals}

Édition électronique

URL : https://journals.openedition.org/tourisme/4097

DOI : $10.4000 /$ tourisme.4097

ISSN : 2492-7503

Éditeur

Association Mondes du tourisme

\section{Référence électronique}

Prosper Wanner, « Observation d'une Venise confinée. Le tourisme, pharmakon vénitien, à la fois remède unique, poison addictif et bouc émissaire », Mondes du Tourisme [En ligne], 20 | 2021, mis en ligne le 15 décembre 2021, consulté le 17 décembre 2021. URL : http://journals.openedition.org/ tourisme/4097 ; DOI : https://doi.org/10.4000/tourisme.4097

Ce document a été généré automatiquement le 17 décembre 2021.

\section{cc) (†) $\ominus$}

Mondes du tourisme est mis à disposition selon les termes de la licence Creative Commons Attribution - Pas d'Utilisation Commerciale - Pas de Modification 4.0 International. 


\title{
Observation d'une Venise confinée. Le tourisme, pharmakon vénitien, à la fois remède unique, poison addictif et bouc émissaire
}

\author{
Observation of a Venice in Lockdown. Tourism, Venetian Pharmakon, Both \\ Unique Remedy, Addictive Poison and Easy Scapegoat
}

Prosper Wanner

1 Venise a été l'une des premières villes en Europe à être confinée. La marée historique de novembre 2019 a vidé la ville des touristes puis le confinement a obligé l'économie touristique à s'arrêter. L'article propose d'observer ce moment unique de "hors tourisme » sous l'angle de la pharmacologie. La pharmacologie, en biologie, est l'étude de l'interaction entre un produit et un organisme vivant à des fins thérapeutiques. Le philosophe Bernard Stiegler a emprunté la notion de pharmakon à Jacques Derrida, luimême l'empruntant à Platon, et a étendu sa signification pour en faire un outil d'analyse de la société à partir de son ambivalence. Pour le philosophe, «les pharmaka sont donc des remèdes en même temps que des poisons. Et leur niveau de toxicité potentielle est proportionnel à leur efficacité thérapeutique " (Stiegler, 2008, p. 124). L'analogie du tourisme en tant que pharmakon évite de le condamner à l'avance et permet de tenter un bilan pharmacologique de ce moment particulier pour essayer d'en tirer des conséquences thérapeutiques. La cure de désintoxication au tourisme, imposée à Venise offre un moment particulier d'observation de cette ambivalence du tourisme, à la fois remède, poison, drogue et aussi bouc émissaire, avec qui la ville devrait apprendre à vivre "pharmacologiquement». La conclusion élargie les possibles, en interrogeant la capacité du tourisme à détourner l'attention d'autres pharmaka.

2 À travers cet article, je souhaite restituer de l'intérieur, et comme anthropologue, un moment peu documenté d'une ville qui fait déjà l'objet de nombreux travaux de recherche sur son histoire et ses enjeux touristiques (Rabbiosi et Wanner, 2020; Bertocchi, Camatti, Giove et van der Borg, 2020; Salzberg, 2019; Salerno, 2018; 
Zannini, 2014; Brilli, 2008; Costa et Martinotti, 2003; De Rita, 1993). Ce bilan pharmacologique s'appuie sur le premier confinement que va connaître la ville. Alors confiné à Venise, j'ai pu observer in situ les cent jours de ce premier confinement depuis l'arrêt du tourisme en plein Carnaval, le 25 février 2020, jusqu'à la réouverture de la libre circulation entre les régions italiennes le 3 juin 2020 . La ville va alors s'enfoncer dans une crise économique dévastatrice, qui va transformer le cadre de vie des habitants confinés sur place. Le confinement va rendre patent l'ambivalence du tourisme à Venise, à la fois remède espéré, poison redouté et bouc émissaire commode. Après avoir rappelé rapidement le contexte vénitien, la première partie de cet article raconte en texte et en images ce moment hors tourisme, suspendu, à travers les lieux, les personnes et les récits que le confinement va (re)rendre visible: les personnes âgées, la nature, les mendicanti (mendiants), les pendolari (navetteurs) et l'espace public. Puis, avec la réouverture partielle des commerces et des mobilités, le retour des étudiants, des habitants de la métropole et des visiteurs de la région. Ces visiteurs d'un jour étaient pourtant devenus le bouc émissaire d'un surtourisme à combattre. Pour attirer ces mordi e fuggi (ceux qui « mordent et s'enfuient»), dénomination péjorative du tourisme de masse, des entreprises vont chercher à adapter leurs tarifs, services et produits à ces passagers d'un jour, alors que d'autres vont choisir de rester fermés en attendant le retour des touristes.

3 Les effets du surtourisme ayant disparu, la seconde partie raconte ce que le confinement va rendre manifeste des causes de la forte dépendance de Venise au tourisme, jusque-là peu discutées. Cette situation inédite va fragiliser le diagnostic touristique réalisé par la ville pour trouver un remède aux effets du surtourisme, qui était jusque-là communément partagé : celui d'une ville de 50000 habitants assaillie chaque année par 30 millions de touristes. Un récit qui a justifié l'adoption d'une taxe d'entrée dans la ville, reportée sine die avec le confinement. Le travail d'observation croisé avec la relecture des quelques chiffres disponibles raconte une réalité assez différente. En conclusion, à partir des alternatives économiques possibles au tourisme que le confinement va (re)mettre à l'ordre du jour, j'interroge la capacité du tourisme à s'imposer : il n'y aurait pas d'alternative possible, au mieux un aménagement. C'est une invitation à se demander s'il ne faudrait pas simplement apprendre à vivre pharmacologiquement avec le tourisme, à Venise comme ailleurs, ou bien si l'enjeu n'est pas d'appréhender la capacité d'hégémonie du tourisme afin de s'ouvrir à d'autres pharmaka, et donc d'autres possibles à venir.

\section{L'observation en confinement}

4 Le premier confinement de Venise a duré cent jours - du 25 février, date du début du confinement en plein carnaval, au 3 juin 2020, date de réouverture de la libre circulation entre les régions italiennes -, un moment hors tourisme d'une ville où le phénomène du surtourisme a été largement décrit (Rabbiosi et Wanner, 2020 ; Bertocchi, Camatti, Giove et van der Borg, 2020; Salzberg, 2019; Salerno, 2018 ; Zannini, 2014 ; Brilli, 2008). Résidant alors à Venise, j'ai collecté des données lors de marches à pied quotidiennes sous la forme de sons, d'images, d'impressions, de constats et de vidéos, complétées par la lecture des réseaux sociaux et de la presse locale et internationale ainsi que par des conversations avec mon réseau relationnel acquis après plusieurs années en tant que résident. L'observation a porté sur ce qui a 
disparu, ce qui est apparu et ce qui est devenu plus visible avec l'arrêt du tourisme, en particulier les personnes, les lieux et les récits. Cela a permis de documenter des situations de Venise déjà connues mais peu étudiées, faute de données, comme par exemple l'importance de la question foncière dans le surtourisme et l'importance des autres visiteurs dans le flux quotidien. Ces observations ont été croisées avec la relecture de données et d'informations disponibles auprès des institutions et médias.

Photo 1. Venise, Grand canal, Ponte Calatrava

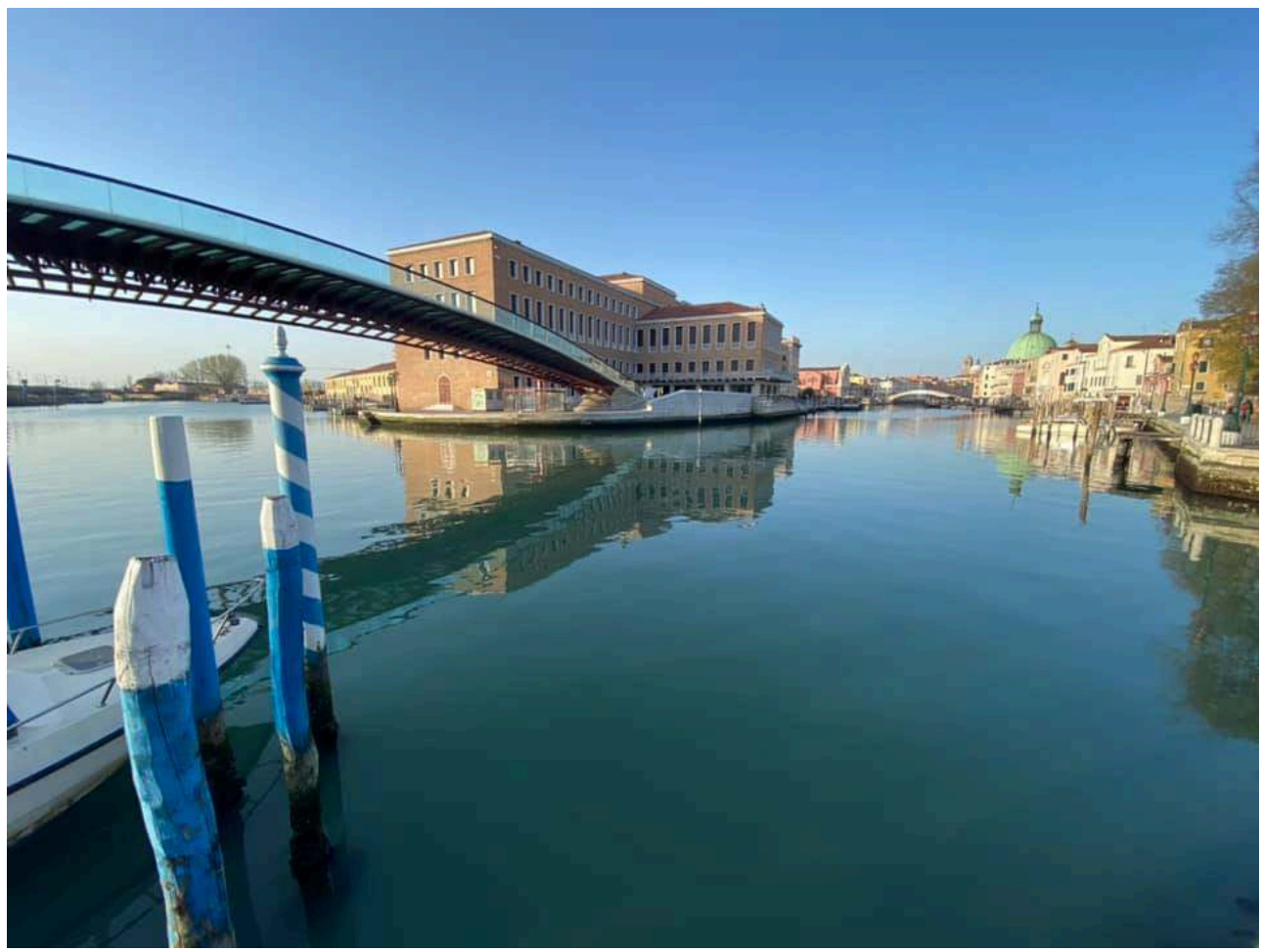

(c) Prosper Wanner, 22 mars 2020

\section{Le tourisme, remède à la désindustrialisation}

Le terme "touriste " provient du "Grand tour », dont Venise faisait partie depuis le $\mathrm{XVI}^{\mathrm{e}}$ siècle avec Naples, Rome et Florence (Brilli, 2008). La vieille ville compte le plus ancien hôtel d'Italie toujours en activité, l'Hôtel Cavalletto e Doge Orseolo de Venise, né comme auberge en 1200. Les archives font mention, dès 1335, d'une organisation des aubergistes alors dénommés cameranti. Les voyageurs étaient alors des diplomates, des marchands, des marins, des pèlerins ou des réfugiés, comme ceux qui fondèrent le ghetto, ou San Lorenzo degli armeni (Salzberg, 2019). Venise fait partie des premières villes en Europe à avoir su développer l'industrie du tourisme. En 1850, le train arrive à Venise et l'administration autrichienne, qui domine la ville de 1815 à 1866, décide d'exploiter son potentiel balnéaire. Par la suite, les stabilimenti balneari (établissements balnéaires) quittent le centre historique pour s'implanter au Lido (Levantis, 2007). La période touristique du novecento veneziano commence avec la fondation de la Compagnie des auberges du Lido en 1906, qui deviendra peu après la Compagnie italienne des grands hôtels qui existera jusqu'à la Seconde Guerre mondiale, suite à laquelle une 
nouvelle phase commence: l'ère du "tourisme de masse", le tourisme devenant l'activité économique prédominante à Venise (Zannini, 2014).

Durant le novecento et jusqu'après la Seconde Guerre mondiale, Venise était avant tout un important port industriel qui comptait 150000 habitants et 70000 postes de travail liés directement ou indirectement à l'industrie. Les activités étaient diverses, liées au tabac, au coton, à la construction navale, à la fabrique de pâtes, de bière, d'horloges, de savons mais aussi à l'industrie du cinéma. Dans la seconde partie du $\mathrm{xx}^{\mathrm{e}}$ siècle, les activités industrielles vont décroître ou se déplacer vers la terra ferma (terre ferme), à Porto Maghera. À la fin des années 1960, plus de la moitié des résidents du centre historique vivaient dans des maisons sans salle de bains, sans chauffage et sans accès à un réseau d'égouts efficient (Favero, 2014). Une partie des habitants part alors sur la terra ferma pour travailler et se loger dans des maisons modernes. Depuis les années 1950, le «centre historique » a perdu $70 \%$ de sa population, qui était alors de 175000 habitants. Paradoxalement, l'arrêt brutal du tourisme rappelle à certains résidents les années 1990, cette époque d'entre deux, entre la fin du déclin industriel et le plein essor de l'économie touristique. Lors de plusieurs conservations avec des résidents ayant grandi à Venise durant cette période, ceux-ci m'ont raconté que, lorsqu'ils étaient jeunes, ils n'avaient le choix qu'entre un ou deux bars pour se retrouver tard le soir. Pour sortir de cette crise industrielle, Venise va tirer profit du tourisme pour la relance économique, culturelle et sociale de la ville. Elle va notamment obtenir en 1987 son inscription sur la Liste du patrimoine mondial de l'Unesco. En 1961, le centre-ville historique (centro storico) comptait 138000 résidents pour 1,7 millions de présences touristiques (nuits sur place); en 1991, il comptabilisait 75000 résidents pour 2,6 millions de présences touristiques (Zannini, 2014; Favero, 2014 ) et en 2019, il comptait 52143 résidents pour 12,9 millions de présences touristiques ${ }^{1}$.

\section{Le tourisme, poison économique}

7 Plusieurs études témoignent de l'évolution de la ville vers une «monoculture touristique » (Costa et van der Borg, 1988), aboutissant à une situation où la population serait « divisée entre ceux qui vivent du tourisme et ceux qui voient le tourisme réduire leurs espaces vitaux» (De Rita, 1993), ce qui amène parfois à qualifier la situation d'hypertourisme (Salerno, 2018) :

du point de vue sociodémographique, il y a hypertourisme chaque fois que les usagers ou les visiteurs d'une ville excèdent le nombre de résidents et, de ce fait, la ville est définie comme un lieu à vocation touristique [...] semblable à un parc d'attraction. (Costa et Martinotti, 2003, p. 61) ${ }^{2}$

Dès 1992, l'Unesco avertit la ville que la « croissance du tourisme de masse ${ }^{3}$ » constitue une menace pour la ville et son environnement. L'organisation avait donné à la ville jusqu'en 2019 pour prendre les mesures nécessaires afin de ne pas entrer dans la liste des «sites à risque ». Cette échéance a finalement été reportée. En 2018, les données sur le tourisme publiées par la région Vénétie comptabilisent, dans la métropole de Venise, 9,6 millions d'arrivées et 36 millions de présences, comprenant l'ensemble des visiteurs, dont les touristes ${ }^{4}$. L'offre d'hébergements touristiques continue de croître : en 2019, l'hôtellerie propose 32523 lits, dont 967 lits supplémentaires, et les structures extra hôtelières proposent 48441 lits, dont 7142 lits supplémentaires $(+17 \%)^{5}$. La croissance 
constante du nombre de visiteurs s'expliquerait, pour partie, par la multiplication des accès à la ville. La fréquentation de l'aéroport international Marco Polo est passée de 4 millions de passagers en 2000 à plus de 11 millions en 2018, avec une croissance moyenne du nombre de passagers de plus de $7 \%$ sur les cinq dernières années. Les compagnies aériennes à bas coût basées à Venise jouent un rôle majeur dans cette augmentation. Six cent bateaux de croisières font en outre escale chaque année à Venise, avec en moyenne 1,5 millions de croisiéristes sur les dix dernières années selon les données 2019 du Terminal passagers. Venise est devenue le symbole d'un surtourisme dont la critique est largement documentée (Urbain, 1991; Lanfant, 2004 ; Tossot, 2007 ; Cousin et Réau, 2011 ; Gravari-Barbas et Jacquot, 2018 ; Christin, 2018).

\section{La désintoxication forcée au tourisme}

9 Avec le confinement, les commerces de proximité ont perdu leur clientèle touristique ainsi que celle des restaurants, bars et hôtels, fermés pour cause de confinement, qui leur achetaient le pain, le poisson, les fruits et les légumes. Plusieurs commerces se sont mis à livrer les habitants : les poissonniers, les libraires, les quincailliers, les papeteries, les boulangers, les supérettes et, comme toujours, les pizzérias. Avant, seules les pizzérias proposaient la livraison à domicile, parfois les vendeurs de journaux ou certains producteurs de la lagune. Dans la cité lagunaire, où les livraisons se font à pied ou en barque, il n'y a pas de livreurs Uber Eats ou Deliveroo qui sillonnent la ville. Seuls les paquets Amazon sont devenus dernièrement bien visibles sur les barques des transporteurs, dans les charriots des livreurs et aux points de livraison ouverts dans quelques commerces. La livraison à domicile des personnes âgées était parfois l'affaire des badante, des aides à domicile, déclarées ou non, originaires en partie des pays de l'Est de l'Europe. Certaines personnes âgées ont logé sur place leur badante durant le confinement. D'autres ont perdu cette aide précieuse dans une ville où les courses se font souvent quotidiennement pour éviter de porter des sacs trop lourds. À Venise, il n'y a pas d'ascenseurs et beaucoup de ponts. Au marché du Rialto, à côté de la banderole "Rialto no se toca» (pas touche au Rialto) qui alerte sur la possible fermeture du marché aux poissons, apparaissent des annonces pour la livraison à domicile. Partout en ville, des volontaires livrent les personnes âgées et celles en quarantaine. J'ai croisé parfois ces jeunes volontaires avec plusieurs listes de courses en main. J'ai aperçu aussi la protection civile qui sonnait aux portes pour livrer des masques, certainement aux personnes fragiles. Il m'est aussi arrivé de croiser un ami libraire et élu de l'opposition (lista civica Casson) qui transportait dans chaque main des sacs de livres à livrer à ses clients. C'était certainement pour lui le moyen de continuer d'exercer son métier et de faire de la politique en temps de confinement. Ces commerçants de proximité, menacés de fermeture à cause du surtourisme du fait de l'augmentation des loyers et de la baisse de la clientèle de proximité, sont devenus des commerçants de première nécessité et se sont mis à assurer le dernier kilomètre de livraison. La livraison des habitants, qui existait dans d'autres villes avant la crise sanitaire, est devenue à Venise une nécessité, tant pour les habitants que pour les commerçants, à nouveau solidaires. 
Photo 2. Venise, San Marco, promeneur

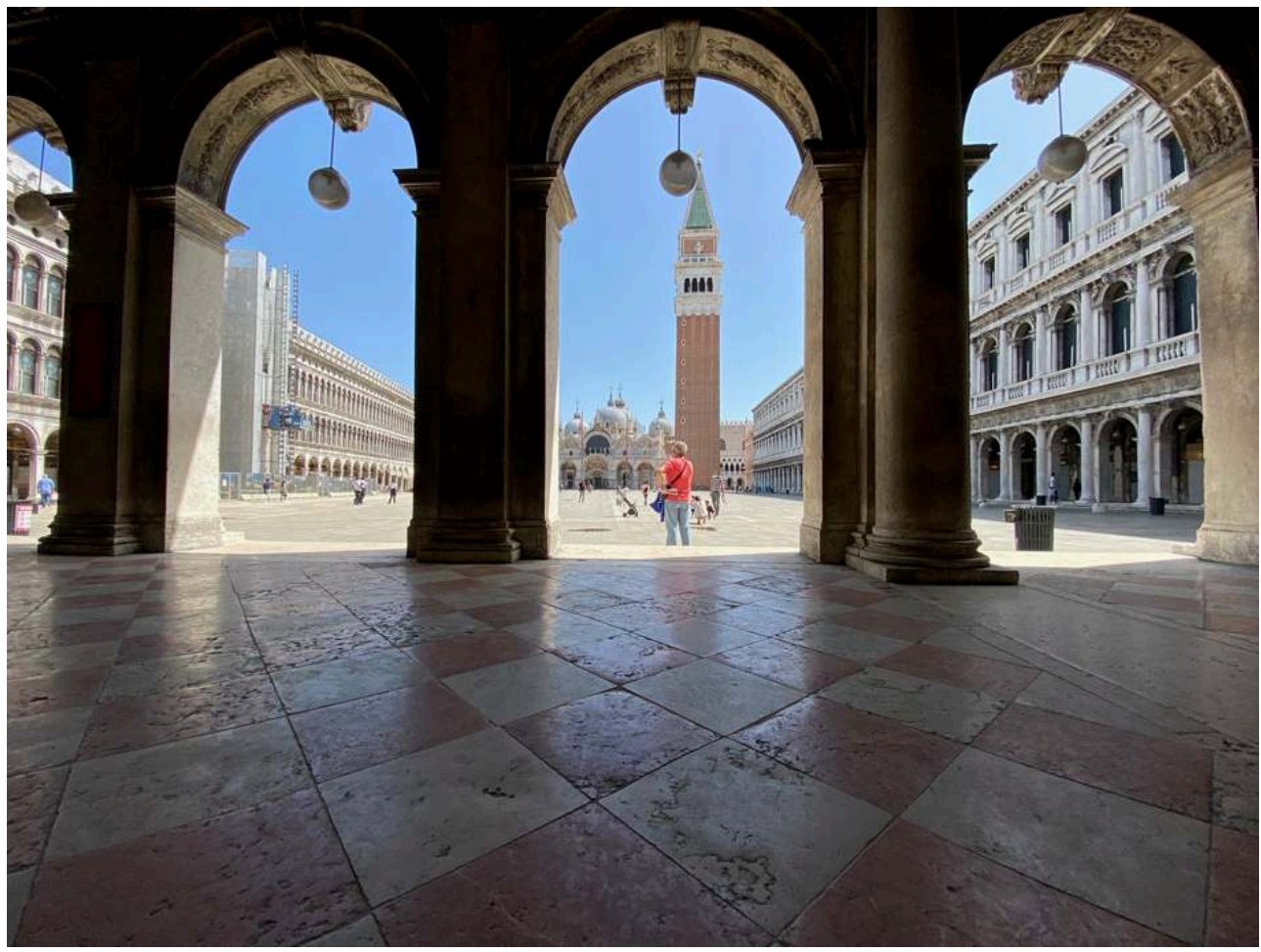

(C) Prosper Wanner, 7 mai 2020

10 Au-delà des résidents, ces commerces et services publics ont continué à fonctionner pour les pendolari (navetteurs), dont une bonne partie n'est pas issue du secteur touristique, ces employés qui viennent chaque jour à Venise travailler dans les administrations publiques, les universités, à l'hôpital, dans les prisons, pour des professions libérales et des cabinets d'avocats ou encore dans les chantiers de restauration. Avec les mesures de distanciation sociale, leur présence est devenue particulièrement visible à Piazzale Roma avec l'arrivée de bus et de vaporetti remplis de personnes portant masques et gants. Ils effectuent leurs trajets entre leurs emplois à Venise et la terra ferma où ils résident. Les journaux titraient sur ces travailleurs obligés de s'entasser dans des vaporetti malgré l'épidémie. Hors période de confinement, les touristes ont peu connaissance de ce flux de personnes car ils se promènent généralement après que les écoliers sont partis à l'école et que sont arrivés les employés, leurs guides et leurs marchandises, après qu'est livrée leur alimentation et que sont ramassés les ordures et leurs draps pour être lavés dans les blanchisseries industrielles à Maghera. Cette invisibilisation des travailleurs permet de mieux comprendre la surprise de certains touristes, avant le confinement, quand ils semblaient découvrir que la ville est habitée : ils se mettaient à prendre des photos des éboueurs, des enfants qui jouent sur les campos et des ambulances sur le grand canal. Le tourisme semble avoir cette capacité à rendre invisibles les autres et à s'émerveiller de leur présence. Avec l'épidémie et les mesures de distanciation, le nombre de travailleurs hors tourisme a fini par faire la une des journaux locaux et à être visible.

Lors de mes sorties, j'ai également été amené à croiser des personnes qui mendiaient de quoi manger. La présence à Venise de mendiants et de lieux d'accueil est ancienne, comme en témoignent les dénominations de l'Église San Nicolò dei Mendicoli et de 
l'Église San Lazzaro dei Mendicanti situées au rio dei Mendicanti. Venise a eu jusqu'à 300 scuole grandi et minori, des confréries laïques qui, sous le patronage d'un saint protecteur, menaient des actions de solidarité et de charité envers les plus faibles et les plus démunis. Elles sont à l'origine de la construction d'hospices et d'hôpitaux. Mais, depuis avril 2008, le maire philosophe de Venise, Massimo Cacciari, de centre-gauche, a interdit la mendicité dans le centre de Venise. «Le phénomène de la mendicité met mal à l'aise les habitants et hôtes de Venise" peut-on lire dans l'arrêté municipal. L'interdiction a pour but d'éviter le " harcèlement » des touristes et la dégradation du « joyau » touristique. Pour le commandant général de la police municipale, il s'agissait aussi de lutter contre un système de mendicité organisé exploitant une centaine de personnes ${ }^{6}$. Pour autant, la mendicité organisée n'a pas disparu avec l'arrêté municipal de 2008 et, jusqu'au confinement, elle est restée très présente. Les mendiants étaient essentiellement des jeunes hommes, réfugiés africains et parfois roms. Chacun avait un emplacement très précis, au centimètre près, où il faisait la manche chaque jour : les ponts, les sorties des supérettes ou d'églises et les sottoportegi (passages couverts). Avec le confinement et la disparation de cette mendicité organisée, j'ai pu observer une autre mendicité. Ce sont essentiellement de jeunes personnes, en détresse, souffrant visiblement d'addiction, qui semblent errer dans la ville. La première fois, j'ai croisé un jeune homme avec un énorme sac à dos et une bouteille d'alcool en main, devisant avec une petite statue de la vierge incrustée dans un mur, comme il y en a tant à Venise. Une autre fois, c'était une jeune fille autour de la vingtaine, le teint blafard, qui arrêtait les passants au marché du Rialto pour leur demander des sous pour manger. En allant tôt au marché, j'ai également croisé une personne âgée avec son caddie, comme il y en a beaucoup à Venise ; en passant à côté d'une poubelle publique, elle a pris le temps de regarder s'il n'y avait pas quelque chose dedans. Des actions de solidarité et de charité ont commencé à être organisées pour secourir les plus faibles et les plus démunis. Un vénitien a fait don d'une somme d'argent à un collectif d'habitants pour aider des personnes en difficulté et la ville a annoncé la mise en place de bons d'aide pour les plus démunis. Ces rencontres m'ont amené à me demander où étaient passés les mendicanti d'avant le confinement et d'où venaient les mendicanti actuels. J'ai reçu quelques témoignages sur le fait que des travailleurs étrangers étaient repartis dans leurs pays faute de travail ou par crainte de l'épidémie alors fortement présente à Venise. Les travailleurs du secteur touristique ont été particulièrement impactés, notamment ceux qui n'étaient pas déclarés? 
Photo 3. Venise, Campo San Giacomo dall'Orio, banc public

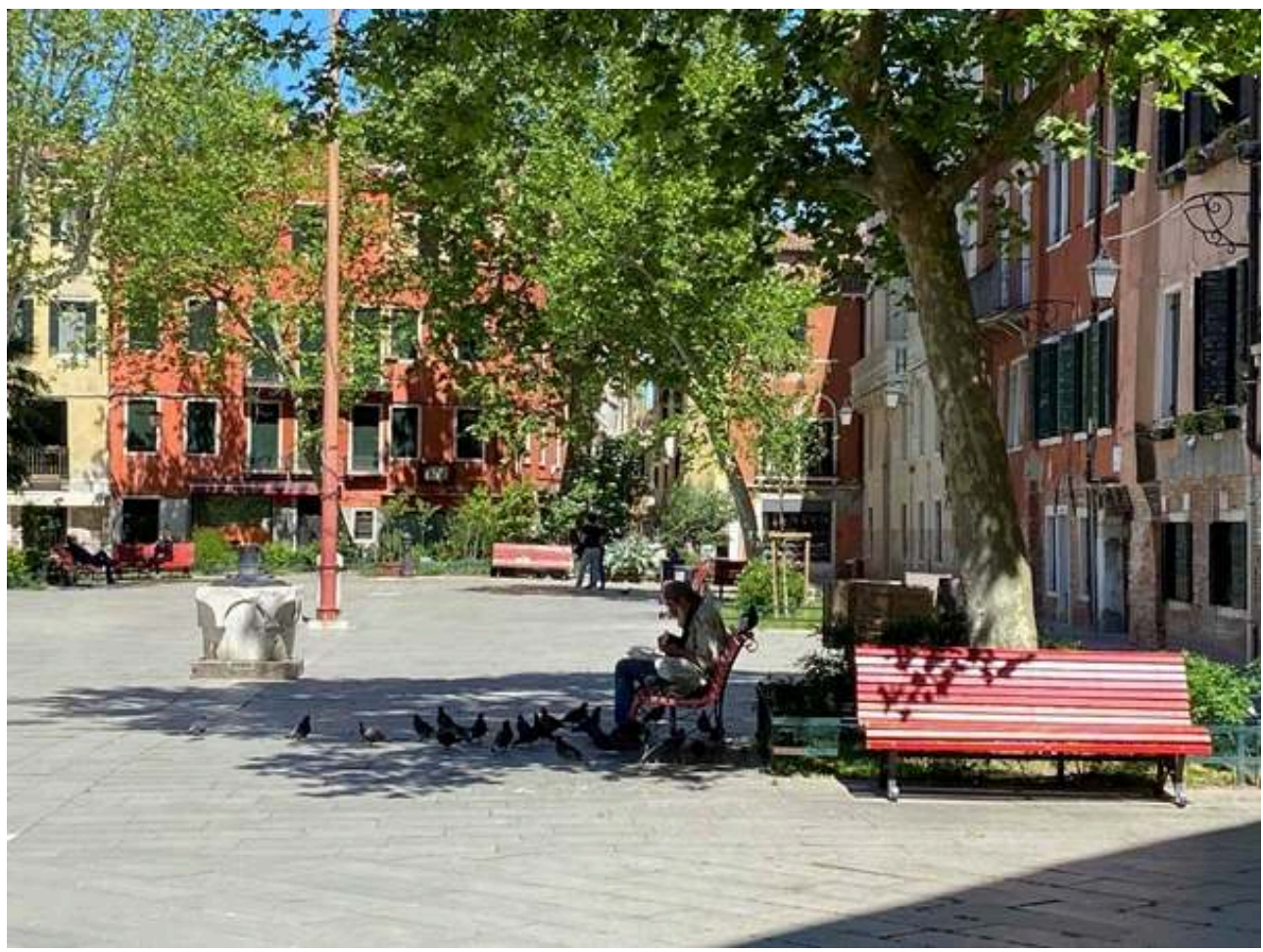

(c) Prosper Wanner, 8 mai 2020

12 Avec l'arrêt des flux touristiques, le son du tourisme s'est tu. Je n'entends plus de ma fenêtre que le seul chant des oiseaux, et non plus celui des avions au loin, la sirène des bateaux de croisière et, plus proches, les annonces de la gare sur l'arrivée et le départ des trains. Cela fait plusieurs fois que je vois de ma fenêtre passer un oiseau de grande taille au vol élégant et au plumage blanc et noir avec un long bec courbé noir. Difficile de dire si c'est un ibis sacré car ils ne seraient qu'une soixantaine dans la lagune. Les oiseaux sont très présents à Venise, encore plus depuis le confinement. Le vacarme du jeune cormoran qui loge juste au-dessus de mon appartement me le rappelle chaque jour. Des canards habitent certains canaux et y promènent parfois leurs canetons. Sur la Piazzale Roma désertée, un couple de canards s'est installé à côté du tabac. Je les ai rencontrés en train de discuter avec le gérant, apparemment habitué à leur visite. Les cormorans ont perdu comme source de nourriture d'abord les poubelles extérieures en 2019 et maintenant les touristes qui faisaient régulièrement déborder les rares poubelles publiques de la ville. Il n'est pas rare de voir ces cormorans chasser les pigeons ou les parts de pizza dans les mains des passants. D'autres oiseaux sont plus visibles dans la ville: les petites mouettes à tête noire ou blanche, les hérons, les aigrettes et de petites poules d'eau aux yeux rouge vif qui chassent dans les canaux. Selon l'Atlante della Laguna (l'atlas de la lagune), qui recense depuis plus de vingt ans la faune et la flore lagunaire, la lagune compterait un demi-million d'oiseaux, avec une population qui ne cesse de croître. Ils seraient cent mille de plus en cinq ans selon le dernier comptage de l'Atlante della Laguna. Les fenicotteri, les flamants roses, se sont installés depuis quelques années dans les vale da pesca nord (vallées de pèche). Ils étaient presque vingt mille en 2019 , soit le double de l'année précédente. Il y aurait autour de 130 espèces différentes d'oiseaux. Les hirondelles sont les plus nombreuses avec un cinquième des présences. Pendant le confinement, il suffisait, pour s'en rendre 
compte, de regarder par la fenêtre ou d'ouvrir sa fenêtre à cinq heures du matin. Un collectif d'acteurs de l'écotourisme faisait la promotion à l'échelle lagunaire d'un tourisme lent dans la lagune, à la découverte de sa flore et de sa faune. L'arrêt brutal du tourisme, et surtout des sorties scolaires, a eu raison de cette initiative. L'arrêt du tourisme semble favoriser le retour dans l'espace public d'humains comme d'autres êtres vivants jusque-là invisibilisés.

Ce retour de la nature en ville a été remarqué, voire attendu. En mars 2020, la vidéo de dauphins nageant dans les eaux de Venise est devenue virale au point d'être retweetée plusieurs centaines de milliers de fois, allant même jusqu'à faire la une de certains médias ${ }^{8}$. Si cette vidéo est une fake news, les dauphins ayant été filmés dans un port de Sardaigne, les chats, tout comme les oiseaux, sont aussi devenus plus visibles. Les chats domestiques ont profité du confinement pour sortir sur les campi. Les chats ont été, par le passé, très présents dans les rues de Venise. Ils servaient à protéger les cargaisons des rongeurs, comme l'exigeaient les assureurs et prêteurs dès le XIII ${ }^{e}$ siècle. Ils ont aidé à lutter contre les invasions de rats et les pestes. En 1989, l'administration a décidé de contrôler la présence des chats errants en confiant à l'association Dingo la gestion d'un refuge sur l'île de San Clemente et la réalisation de campagnes de stérilisation faisant passer leur nombre de 12000 à 2000 dans la lagune ${ }^{9}$. Les chats se sont faits plus rares, sans pour autant quitter complétement la ville. Il y a celui du libraire qui trône à côté de la caisse enregistreuse, celui du menuisier qui monte la garde devant la porte, celui de la potière qui regarde par la vitrine les passants et ceux de l'hôpital qui dorment paisiblement dans le cloître. Il existe encore quelques parcs ou calle (rue) peu fréquentés où l'on peut voir encore des cabanes où des chats dorment paisiblement à côté de gamelles encore remplies de nourriture. C'est surtout dans les îles de la lagune que l'on peut encore croiser des chats errants, parfois en nombre. Avec le confinement, les chats ont semblé sortir des appartements ou de leurs cachettes et réinvestir l'espace public. Les habitants les sortent devant leur porte et parfois les emmènent faire un tour. À chacune de mes sorties, je rencontre ces chats, à côté de leurs maîtres qui discutent. Ils se mettent au soleil, observent la rue. Avec le départ des touristes, l'espace public ne semble plus leur être interdit. 
Photo 4. Venise, Castello, abri pour chats

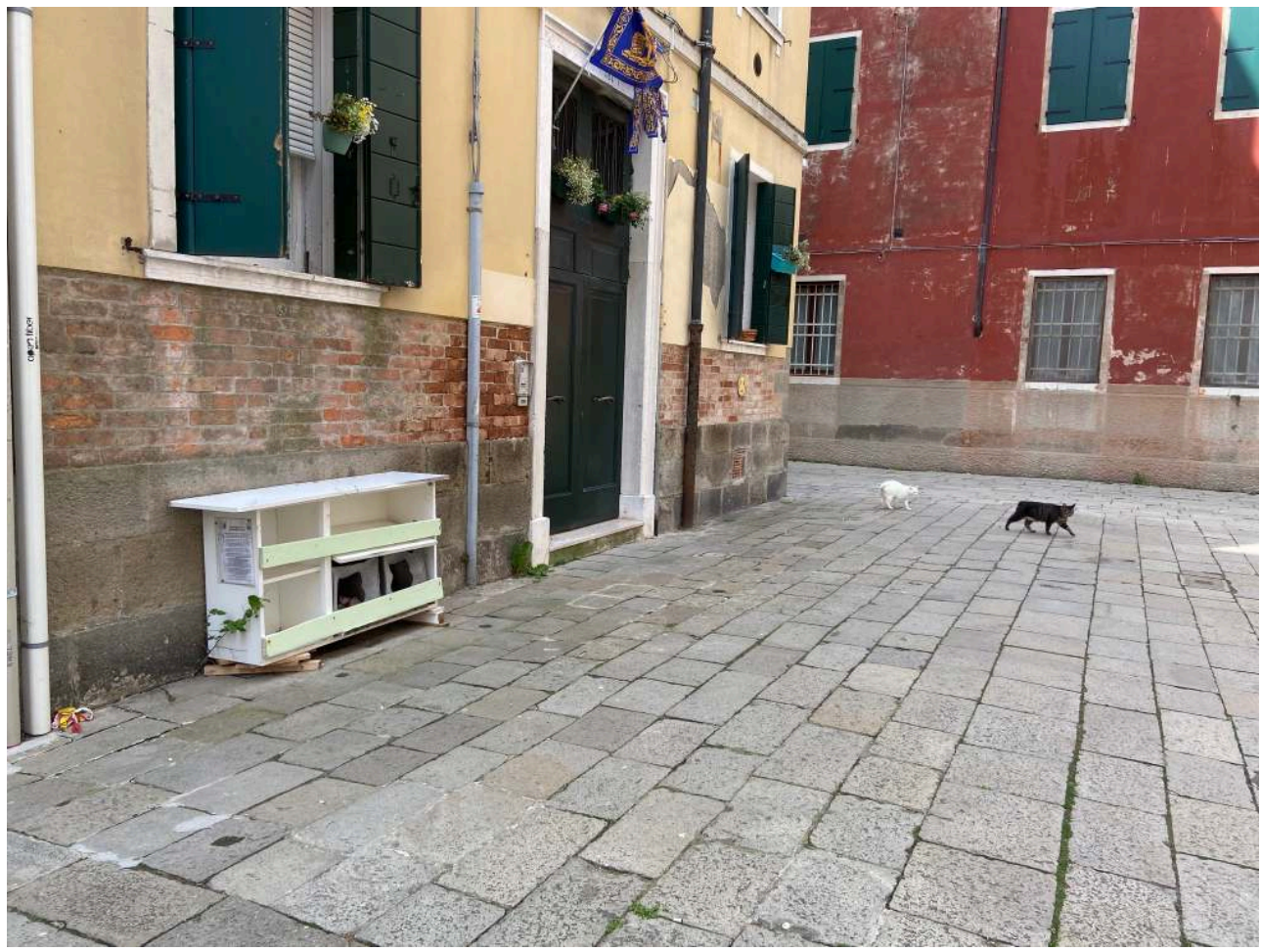

(c) Prosper Wanner, $7^{\text {er }}$ mai 2020

Au-delà des espaces publics libérés des touristes et des étalages de souvenirs, d'autres lieux ont été à nouveau accessibles, comme les églises. Avec le confinement, il a été possible d'entrer librement dans les églises sans payer ou prouver sa résidence. Elles semblent revenues à une certaine normalité. Un dimanche, nous étions seuls dans la basilique San Marco. L'entrée principale était maintenue fermée et l'entrée se faisait par le côté. Seule une partie de l'église était accessible. À l'entrée, il fallait se laver les mains au gel hydroalcoolique ou bien mettre des gants. Un panneau précisait que l'église était ouverte le matin "pour se recueillir ». Quelques chaises permettaient de s'asseoir face à la cappella della Madonna Nicopeia. Le reste de l'église était désert. Le silence régnait parmi les riches mosaïques au sol et celles d'or couvrant murs et plafonds. 
Photo 5. Basilique San Marco, cappella della Madonna Nicopeia

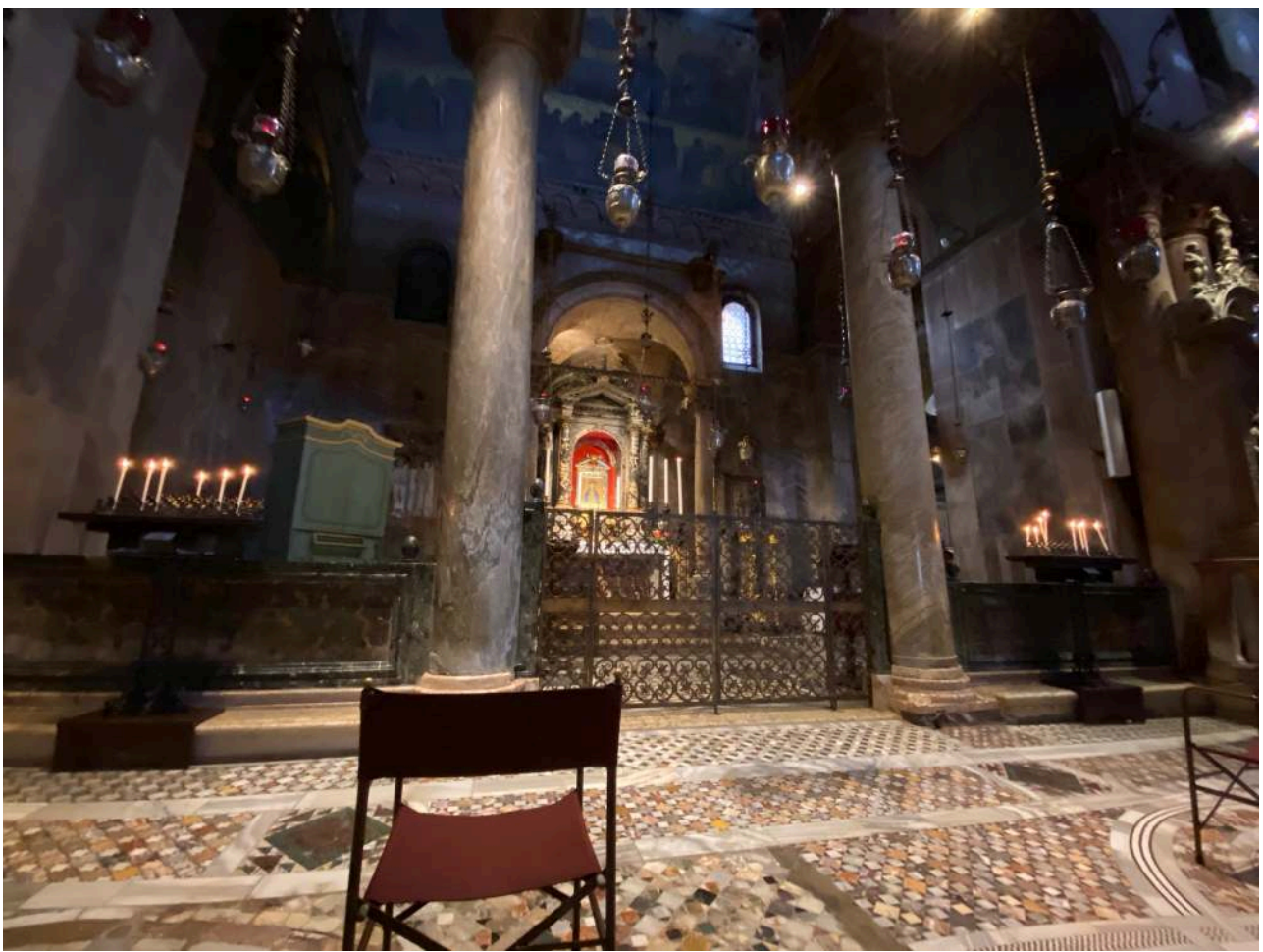

(c) Prosper Wanner, 17 mai 2020

Ces observations sont visibles à travers les nombreuses images qui ont circulé, au niveau local comme international. Les photos des eaux limpides des canaux vénitiens et des places désertées, en particulier celles normalement envahies par les touristes, ont circulé dans les médias et les réseaux sociaux. Venise est certainement l'un des lieux au monde dont les images sont le plus partagées. Les habitants ne savent pas sur combien de photos leur image a été capturée par mégarde. Si les Vénitiens partagent aussi des photos de leur ville, ce sont souvent des photos de grands événements symboliques pour la ville, comme la fête du Redentore ou celle de la Salute, qui témoignent de leur présence et de leur attachement à ces événements. Un groupe Facebook rassemble ainsi plus de 70000 membres, dont de nombreux Vénitiens, qui partagent des photos de lieux non touristiques de Venise et de sa lagune. Le règlement du groupe précise que ne sont pas acceptées les photos de la place San Marco, du Rialto et de la Punta della Dogana. Chacun peut y poster sa photo d'un lieu méconnu ou une image d'archives. À chaque nouvelle photo postée, les membres du groupe essaient de deviner l'endroit et les photos reçoivent des centaines de like. De manière générale, les Vénitiens partagent rarement des photos des lieux touristiques, sauf pour témoigner de leur surfréquentation ou pour dénoncer les comportements des visiteurs qu'ils jugent inopportuns. Avec le confinement, les Vénitiens se sont mis à partager fréquemment leurs photos de ces lieux très touristiques, comme San Marco, et de lieux communs, comme la gare, les canaux et la Strada Nova. Ces photos témoignent du calme de l'eau et du vide de la ville, mais aussi d'une beauté retrouvée comme en témoignent les commentaires associés aux photos. Ce sont les lieux auparavant surfréquentés par les touristes qui semblent aujourd'hui fasciner les Vénitiens: San Marco, le Rialto et le grand canal. Comme si les habitants voulaient graver de manière durable l'image de ces lieux emblématiques abandonnés des touristes et du tourisme pour cause d'épidémie. 
16 Avec la mise en place du confinement, Venise se retrouve hors tourisme: sans ses stands de souvenirs, ses guides touristiques, ses transports touristiques, ses restaurants et résidences touristiques. Seuls restent visibles quelques vitrines éteintes et de grands panneaux informatifs. Pour l'historien Marc Boyer, « aucun lieu, aucun monument n'a en soi vocation touristique. Il faut le verbe des hommes" (Boyer, 1999, p. 97); puis l'existence sur place de guides, d'hôtels et de restaurants. La Venise touristique semble devenue muette. Personne n'est plus là pour la raconter et l'écouter. Les guides ne répètent plus à l'infini le grand récit de la Sérénissime à des groupes de visiteurs équipés d'écouteurs portables. La ville n'est pour autant pas vide de personnes ni de récits.

\section{Le touriste, bouc émissaire commode}

Durant le premier confinement, avec la réouverture partielle des commerces et des mobilités le 11 mai 2020, des visiteurs vont à nouveau venir en ville, notamment les étudiants et les campagnoli, ces habitants de la métropole et de la région. Ces visiteurs d'un jour étaient pourtant classés parmi les mordi fuggi, dénomination locale péjorative pour désigner le tourisme de masse. Cette dénomination comprend à la fois les visiteurs lointains des croisières ou des voyages en groupes et ceux plus proche des grandes stations balnéaires ou de la région Vénétie. Ces mordi fuggi étaient devenus le bouc émissaire d'un surtourisme à combattre. Avec le retour des campagnoli, certaines entreprises vont adapter leurs tarifs, services et commerces pour ces visiteurs d'un jour, comme pour les habitants et les pendolari. La ville va alors compter sur ses 270000 résidents, campagnoli compris, et ses 20000 étudiants pour faire marcher son économie. Une association d'hébergeurs individuels va établir un partenariat avec l'université pour accueillir les étudiants dans leurs hébergements inoccupés. En mai 2020 , la ville va passer un accord avec les 250 bateaux taxis afin de proposer, durant un mois, des tarifs spéciaux aux habitants ${ }^{10}$. Le tarif proposé était de 20 euros pour un taxi à plusieurs de la Piazzale Roma au Rialto. L'accord a été signé par la commune pour compenser la baisse de $70 \%$ des transports publics en vaporetto. Cette décision fait suite à des articles de journaux relayant les difficultés rencontrées par des habitants devant se rendre régulièrement à l'hôpital. Avec l'absence des touristes, la régie de transport public ACTV a largement réduit ses courses. Elle prévoyait en 2020 une perte de 100 millions d'euros et le préfet avait annoncé que l'entreprise publique risquait de fermer. Venise possède, grâce au tourisme, un système de vaporetto qui fonctionne 24 heures sur 24 . Vous pouvez rentrer d'une île au nord de la lagune à quatre heures du matin, et cela pour 1,40 euros. Cette qualité de service public est rendue possible parce que les touristes payent eux 7,50 euros pour un trajet, soit cinq fois plus que les résidents. L'abonnement mensuel résident est de 35 euros, bus et tramway compris, moins cher que le billet touristique pour trois jours. Grâce à cette politique tarifaire, le tourisme finance un transport public continu à Venise. La contrepartie est une surfréquentation de certaines lignes. Certains embarcadères possèdent une entrée prioritaire pour les résidents afin de leur éviter de rater leur vaporetto, surtout s'ils résident dans les îles de la lagune nord. En dehors de ces embarcadères, il n'est pas rare de rater un vaporetto, voire deux, surtout en été. La marche à pied s'impose alors, même avec un landau ou un chariot chargé de courses. Un autre service public fortement impacté a été celui de la collecte des déchets. Avant le Covid-19, le tourisme 
était accusé de faire exploser les coûts de traitement des déchets, auxquels les entreprises contribuent via une taxe liée à leur volume de détritus, ainsi que les habitants. Le maire s'est servi de cet argument pour justifier la mise en place d'une taxe de débarquement afin que les touristes y contribuent également. Avec la crise, la collecte a baissé de deux tiers et l'absence de tourisme met in fine en difficulté l'entreprise publique dont les ressources financières ont baissé d'un quart. Parmi les mesures prises pour limiter les pertes, mentionnons la réduction du nombre de bateaux de transport des déchets et la fermeture des toilettes publiques jusqu'au retour des touristes. Les résidents et les pendolari ne semblent pas être perçus comme de potentiels usagers de ce service public.

Au-delà du tarif élevé du ticket de vaporetto, les touristes sont largement mis à contribution financièrement. La taxe de séjour est de 4 euros par touriste, la traversée du grand canal en traghetto est de 2 euros pour les touristes contre 0,70 euros pour un résident, l'entrée dans une église est à 2 euros par touriste et gratuite pour un résident, et le tarif du ticket de débarquement a été fixé entre 3 et 10 euros par touriste. Le secteur privé applique également cette double tarification. Le prix ne sera pas le même dans de nombreux commerces de Venise si vous êtes résident, voire si vous parlez le dialecte vénitien, ou non. Pour le maire, réélu au premier tour en 2020, «si vous venez à Venise, vous devez savoir que vous êtes à Venise et dépenser quelque chose ». C'était sa réponse à trois touristes orientaux qui s'étaient plaints auprès de lui d'avoir payé 526,50 euros pour un repas de poisson dans un restaurant vénitien ${ }^{11}$. De plus en plus de touristes, essentiellement asiatiques, partageaient sur les réseaux sociaux les photos des additions des restaurants ou cafés vénitiens tellement leurs montants leur semblaient excessifs. Ces tarifs élevés financent autant les services publics que l'ensemble de la filière touristique, bien au-delà de sa partie visible. Un pâtissier me faisait ainsi part de sa crainte que les intermédiaires profitent de la situation pour renforcer leur position vis-à-vis des bateaux de croisière. Leurs passagers arrivent parfois dans sa boutique lorsqu'ils ne sont pas pris en charge par les nombreux intermédiaires existants. Sa crainte est que, si leur point d'arrivée n'est plus dans Venise, ils soient embarqués via des intermédiaires dans des navettes, par bus ou bateaux, et guidés seulement vers certains commerces, contre commission, une pratique courante à Venise. Le Fontego dei Tedeschi, au pied du Rialto, transformé en énorme supermarché du luxe, a son arrivée directe pour les taxis. Des fabriques de verre de Murano ont elles aussi leurs pontons privés pour y laisser débarquer les taxis ou bateaux-bus chargés de touristes. Cela évite qu'ils passent par les rues commerçantes. En échange, l'intermédiaire, qu'il soit taxi, guide, agence de voyage ou portier d'hôtel, touche une commission, parfois importante. Des magasins ont développé leur propre réseau d'intermédiaires. Le scandale a éclaté au grand jour en 2018 suite au contrôle fiscal d'une dizaine de fabriques de verre de Murano. Les contrôleurs ont mis à jour un vaste système d'évasion fiscale et de caisses noires: le sistema Murano. Ces caisses servaient à payer des intermédiaires, et cela se chiffrait parfois en dizaines de milliers d'euros. Les contrôleurs financiers ont découvert un accord illicite portant sur des millions d'euros entre des verriers de Murano et une agence de voyage internationale. La captation du flux de voyageurs commence dès leur arrivée aux parkings du Tronchetto. Au parking d'arrivée des Pullman, la «mafia du Tronchetto » fait régulièrement la une des journaux. Les touristes y sont incités par des «agents d'accueil » plus ou moins officiels à utiliser les barques de transport privées plutôt que les transports publics. Une pratique tolérée depuis des années qui ferait 
vivre plusieurs familles. Aucun maire n'a mis fin à ce système pourtant maintes fois dénoncé.

Photo 6. Venise, quartier San Marco, rue commerçante

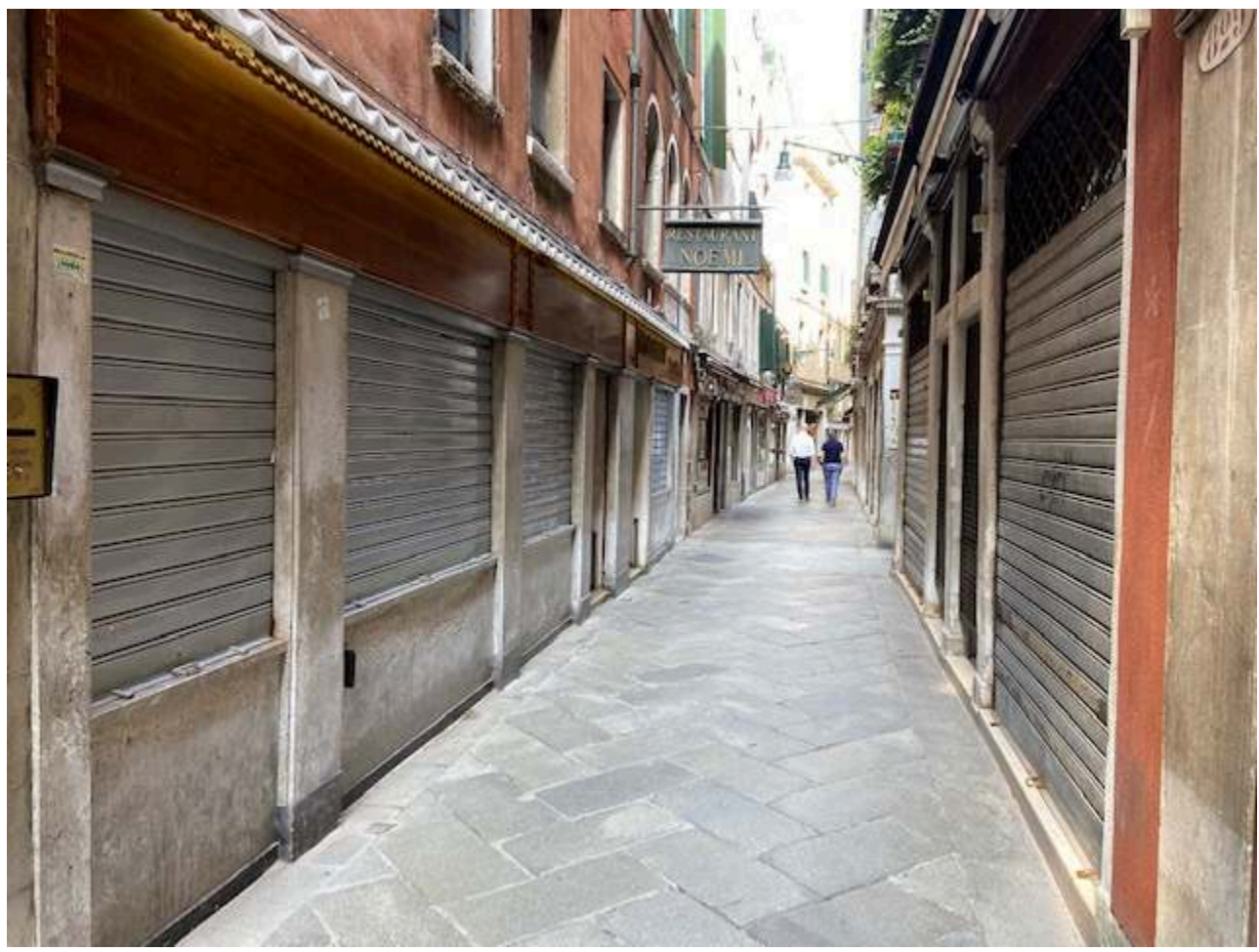

(C) Prosper Wanner, 2 juin 2020

Outre ces intermédiaires, la crise sanitaire a mis en évidence l'importance du rôle des propriétaires dans l'économie touristique. Selon des amis restaurateurs, taxis, agents immobiliers et commerçants, la réouverture des activités dépendra grandement de l'attitude des propriétaires. Des négociations étaient déjà en cours depuis la grande acqua alta (marée haute) de novembre 2019. Même si Venise n'a jamais retrouvé son niveau d'activité commerciale d'avant la crise de 2008, le niveau des loyers reste élevé que ce soit pour se loger ou gérer un commerce. Certains propriétaires ont pris les devants. D'autres accordent de simples délais de paiement ou font la sourde oreille. La logique a plutôt été, jusqu'à la crise, d'augmenter significativement les loyers dès que possible. Il y a deux ans, le gestionnaire d'un café de la place San Marco n'a pas renouvelé le bail d'une partie de son local, que sa famille occupait depuis 67 ans. Son propriétaire lui a doublé le loyer pour arriver à 40000 euros par mois. Depuis quelques années, ce type d'histoires fait régulièrement la une des journaux. Les magasins dits historiques ferment au moment du renouvèlement de leur bail : papèteries, magasins de chaussures, charcutiers, libraires. Le niveau de loyer demandé par les propriétaires augmente tellement qu'il n'est plus tenable pour une activité non touristique. Cela arrive aussi lorsqu'un artisan cherche à céder son activité qui, bien que rentable, ne permet pas d'assumer un loyer important. Pour payer de tels loyers, il faut dégager des marges que seuls le luxe et le low cost rendent possible. Seuls ces produits, à fortes marges, semblent permettre de payer un loyer élevé: d'un côté le luxe avec ses restaurants et commerces, de l'autre le low cost avec la vente à emporter et les souvenirs bas de gamme. Un agent immobilier m'expliquait que, pour une grande 
marque, Venise était une vitrine de luxe avec des millions de visiteurs. Qu'importe si le magasin fait des pertes à cause d'un loyer élevé, le retour en image est sans commune mesure. Les masques et le verre élaborés sur place restent parfois compétitifs. Pour les souvenirs bas de gamme, les commerçants se fournissent chez des grossistes hors Venise, auprès desquels il est possible de trouver à bas prix les masques, le verre, les souvenirs ou des imitations de peintures vénitiennes. Le gestionnaire d'un semigrossiste chinois de Padou racontait ainsi dans un journal travailler davantage avec les magasins dits " historiques " ${ }^{12}$. La famille Cipriani ne rouvrira pas le Harry Bar, fermé pour la première fois depuis 89 ans ${ }^{13}$. Selon cette famille vénitienne millionnaire exilée dans les paradis fiscaux, la faute en reviendrait au manque d'aides publiques et aux contraintes sanitaires.

Photo 7. Venise, San Polo, vitrine « Venise renaît si... elle se défend des spéculateurs »

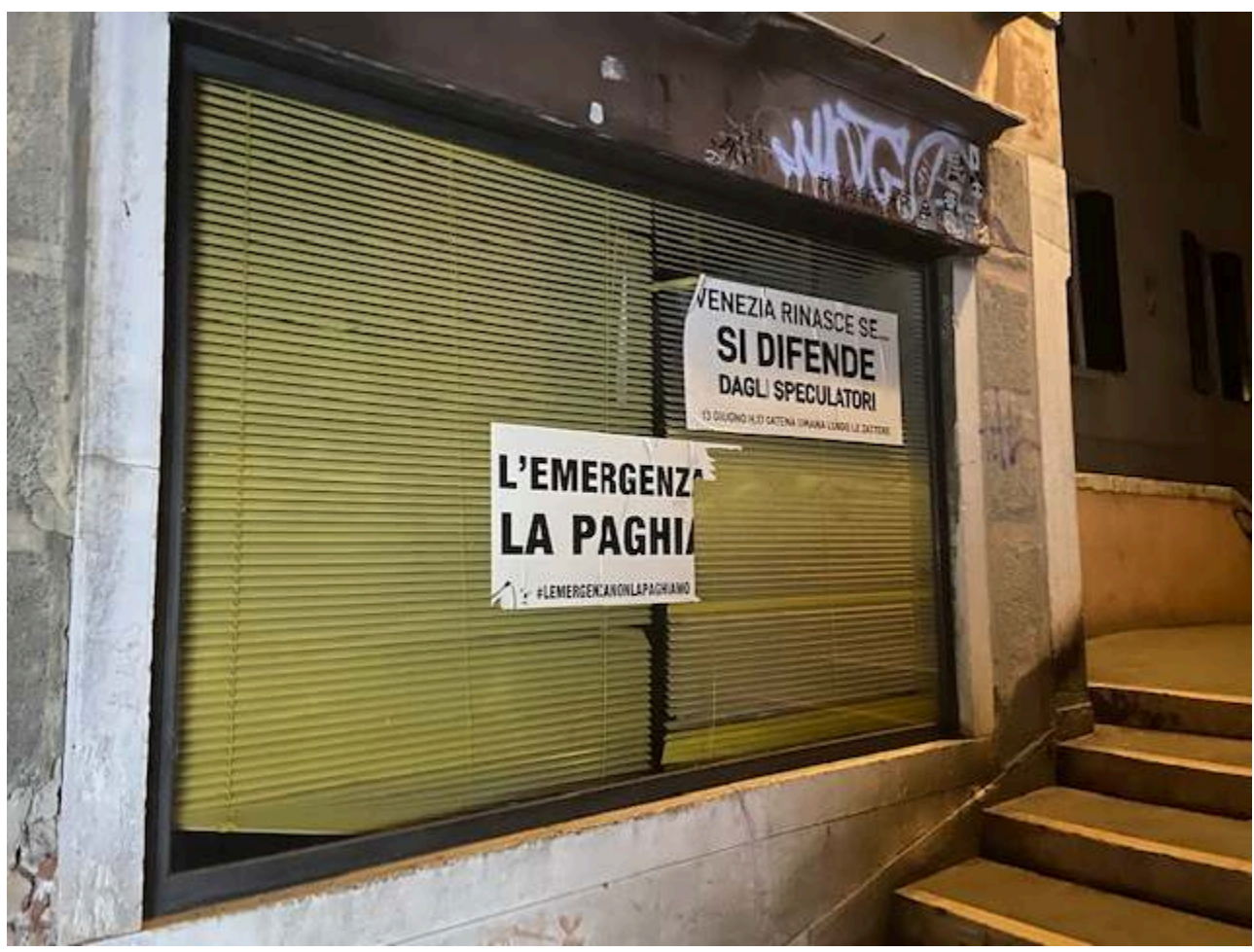

(C) Prosper Wanner, 2 juin 2020

Les loyers d'habitation sont eux aussi tirés vers le haut par la spéculation touristique. Dans les agences immobilières, les appartements sont présentés comme des produits financiers avec leur taux de rendement. En 2018, l'émission «Envoyé spécial» a pu filmer une manifestation de Vénitiens contre l'expulsion d'une retraitée de 70 ans qui payait régulièrement son loyer depuis 52 ans. Le propriétaire, interrogé, et visiblement exaspéré, disait être chez lui et avoir la loi pour lui. L'année dernière, une manifestation similaire a fait reculer un propriétaire qui tentait à nouveau d'expulser une personne âgée. Lorsqu'un bail est renouvelé, qu'un commerçant ou un artisan part à la retraite ou qu'un appartement se libère, le loyer demandé est tel qu'il ne permet souvent qu'une activité touristique. Le groupe citoyen « 25 aprile » a publié un glossaire sur «habiter à Venise». Il est précisé en introduction que "chercher une maison à Venise est maintenant aussi facile que de trouver une pépite d'or sur les rives du Sile, sauf pour les riches ». Sans le tourisme international, il semble difficile de maintenir le 
niveau actuel des loyers. Un couple gérant une quinzaine d'appartements touristiques pour le compte de propriétaires, avec qui j'échange régulièrement dans le cadre de ma thèse, s'est ainsi rapidement trouvé en grande difficulté. Ils ont dû renégocier leurs contrats faute de touriste. Ils proposent aux propriétaires de leur payer un loyer supérieur au marché locatif longue durée ou bien de partager les revenus locatifs touristiques. Dans le premier cas, ils ont pu rendre les appartements aux propriétaires et arrêter de payer les loyers. Dans le second cas, ils ont proposé les appartements à des étudiants pour garder un revenu minimum. La crise sanitaire a mis en avant l'importance de la question foncière, qui semble être l'une des clefs de l'avenir de Venise. Un groupe d'habitants a créé, juste avant la crise, un observatoire du logement et de la résidence à Venise, dénommé OCIO (Osservatorio CIvicO sulla casa e la residenza).

21 Avec le confinement, il n'y a pas que les touristes qui ont déserté la ville et ses espaces publics. Les souvenirs ont quasiment disparu de Venise et, avec eux, les nombreux magasins de souvenirs. Certains commerces de première nécessité semblent aussi avoir baissé le rideau faute de souvenirs à vendre. Cela s'explique par le fait qu'un des moyens de limiter l'invasion des boutiques touristiques à Venise est de réserver certains locaux commerciaux à des activités de proximité comme l'alimentaire ou la vente de journaux. Des commerçants détournent parfois ces règles au profit d'une activité touristique. Ainsi, de nombreux kiosques à journaux se sont transformés en commerces de souvenirs et il est difficile de voir les journaux sous les innombrables masques, sacs et souvenirs qui sont suspendus tout autour. Le règlement, comme le rappellent les quelques articles de journaux à ce sujet, dit qu'ils doivent proposer $a$ minima $55 \%$ de journaux bien visibles et $45 \%$ de souvenirs. Aujourd'hui, les souvenirs ont quasiment disparu des kiosques restés ouverts et les journaux sont redevenus visibles. Une grande partie des stands de fruits et légumes du marché du Rialto ont fermé : ce sont ceux qui vendaient, aux côtés de quelques fruits et légumes, de nombreuses confections d'herbes aromatiques ou de produits séchés pour cuisiner italien. Des supérettes ont aussi fermé leurs portes. À côté des produits du quotidien, elles proposaient des produits à consommer sur place, comme des sandwichs, des boissons individuelles et des souvenirs alimentaires en grande quantité. La plupart de ces commerces étaient tenus par des Asiatiques, bengali ou chinois. Pendant le confinement, ces supérettes auraient pu rester ouvertes - mais apparemment pas sans les touristes. L'arrêt brutal du flux touristique a mis à jour l'ampleur des commerces de première nécessité dépendant in fine principalement du tourisme.

Ces observations amènent à relativiser la seule responsabilité des touristes, seuls ou en groupes organisés, dans la crise que traverse la ville de Venise. Elles dévoilent d'autres causes de la forte dépendance de Venise au tourisme, jusque-là peu discutées, avec un marché foncier injuste, des discriminations tarifaires, des transports publics défaillants, des réglementations détournées, des abus de position dominante, de l'illégalité et des services inadaptés aux habitants. Le succès touristique permet d'augmenter les loyers comme les tarifs des transports en commun, les taxes sur les déchets ou le prix d'un menu gastronomique. Les acteurs publics, privés et illégaux se sont révélés fortement dépendants de cette économie et le «touriste » est devenu un bouc émissaire commode. Le confinement a paradoxalement mis en exergue l'ambivalence de l'économie touristique : la situation est arrivée à un point tel où, avec touristes, les services publics comme les activités touristiques arrivent à saturation, mais sans touristes, il n'y a plus de services publics ni d'économie. Cette crise rend 
visible l'ambiguïté, voulue ou non, qui consiste à ne s'attaquer qu'aux seuls effets du surtourisme, c'est-à-dire les mordi fuggi, plutôt qu'à ses causes. Pour le philosophe Bernard Stiegler, un pharmakon peut aussi jouer le rôle de bouc émissaire :

Poison et remède, le pharmakon peut aussi devenir le bouc émissaire de l'incurie qui ne sait pas en tirer un parti curatif et le laisse empoisonner la vie des incurieux, c'est-à-dire de ceux qui ne savent pas vivre pharmacologiquement. Il peut aussi conduire par sa toxicité à désigner des boucs émissaires des effets calamiteux auxquels il peut conduire en situation d'incurie ${ }^{14}$.

Photo 8. Venise, grand canal

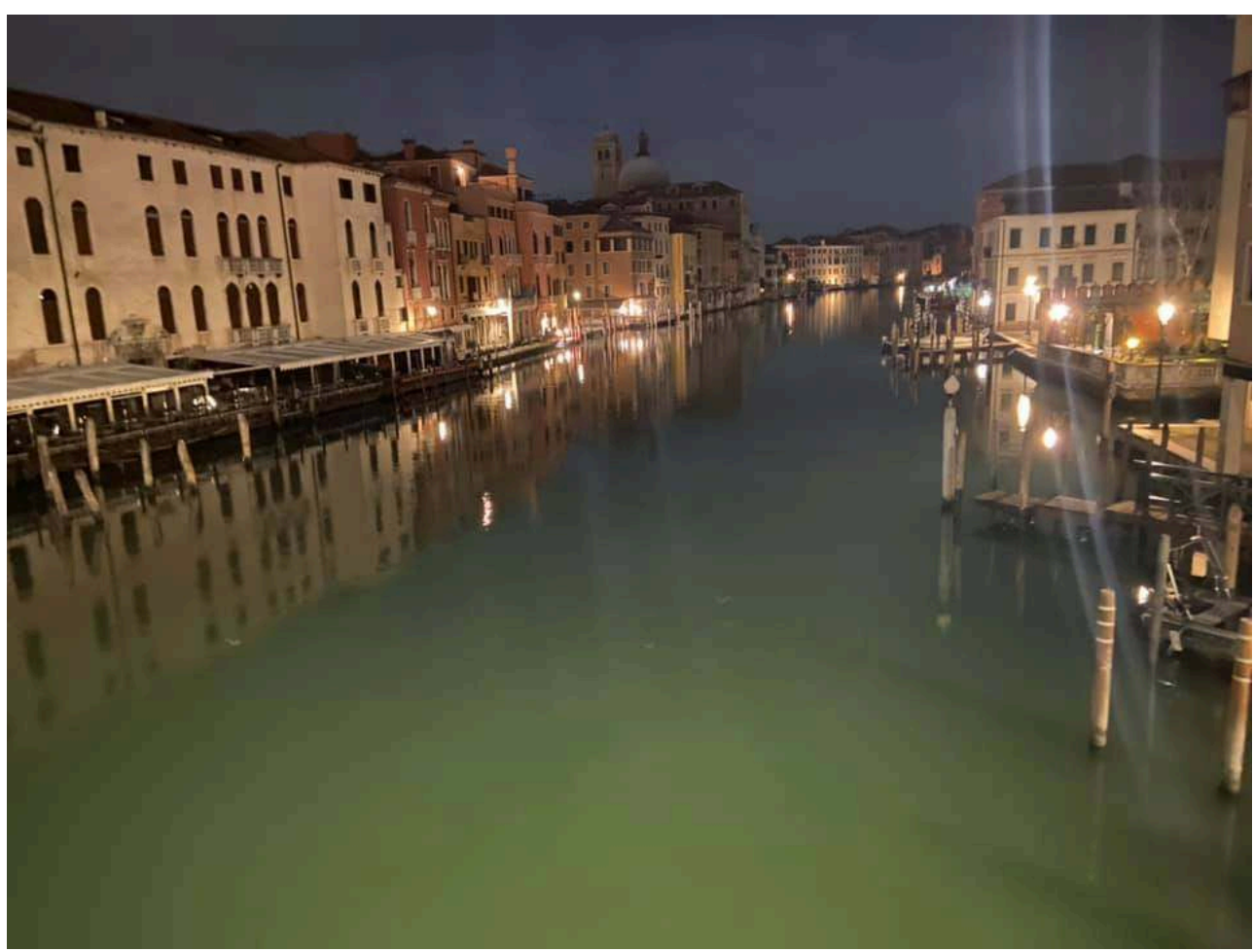

(c) Prosper Wanner, 10 mars 2020

\section{Le diagnostic du phénomène des mordi fuggi}

La taxe de débarquement qui aurait dû s'appliquer dès juillet 2020 n'a pas été mise en œuvre par la ville. Cette taxe dite "de débarquement» a été adoptée par la ville de Venise le 22 novembre 2018 et plusieurs fois reportée au vu de la complexité de sa mise en œuvre. En demandant aux premiers visiteurs de l'après-crise de payer 10 euros chacun, comme cela était prévu en période estivale, la ville aurait certainement freiné la timide reprise économique. Cette décision réinterroge le récit communément partagé par les habitants, les médias et les institutions: celui d'une ville de 50000 habitants assaillie chaque année par 30 millions de touristes. À l'ouverture du Carnaval le 8 février 2020, jour de pic touristique, 100000 visiteurs sont venus, dont 25000 sur la place San Marco, soit un résident du centre-ville pour deux visiteurs. Nous sommes loin du récit médiatique d'un habitant pour 600 touristes. La ville de Venise compte 270000 résidents, dont 55000 résidents dans le centre-ville historique situé au cœur de la Lagune. Elle est la capitale de la région Vénétie, qui compte 5 millions d'habitants. Les 30 millions de présences se divisent grosso modo en 10 millions de 
présences touristes qui dorment en moyenne 2,5 nuits sur place et payent leur taxe de séjour, 10 millions de présences excursionnistes qui visitent la ville en journée et proviennent majoritairement de la région ou des stations touristiques proches et 10 millions de présences visiteurs hors seuls loisirs qui viennent pour les études, le travail, l'administration ou voir des amis (Wanner, 2019). En 2018, l'Université Ca'Foscari a proposé comme modèle durable de fixer un seuil de 19 millions de visiteurs à l'année, soit 52000 présences par jour en centre-ville. Soit un habitant pour un visiteur. Si l'on écoute les arguments de la ville, le problème est qu'un excursionniste le visiteur d'un jour - a un panier de dépenses moyen beaucoup plus faible qu'un touriste qui reste au moins une nuit sur place. L'une des rares enquêtes sur la consommation touristique, réalisée en 2012 par l'Université de Ca'Foscari, révèle que les touristes résidentiels dépenseraient en moyenne 169 euros par personne et par jour à Venise contre 40 euros pour ceux résidant dans des hôtels aux alentours - dit les excursionnistes impropres - et les excursionnistes dits traditionnels 25 euros, soit six fois moins (Van Der Borg, 2013). Et ce alors que le nombre d'excursionnistes connaît une croissance beaucoup plus dynamique. Avec un rapport de $20 \%$ de touristes résidentiels pour $80 \%$ d'excursionnistes, la situation est loin du rapport de 50/50 que préconise l'université (Bertocchi et al., 2020). Avec le confinement, la ville a dû reporter pour la quatrième fois l'application de sa taxe qui devait inverser le rapport entre les touristes résidents et les mordi fuggi. Son objectif, de favoriser les visiteurs dépensiers par rapport aux autres, c'est-à-dire in fine le tourisme international, a été rendu impossible.

Photo 9. Venise, Campo San Giacomo dell'Orio, magasin de souvenirs

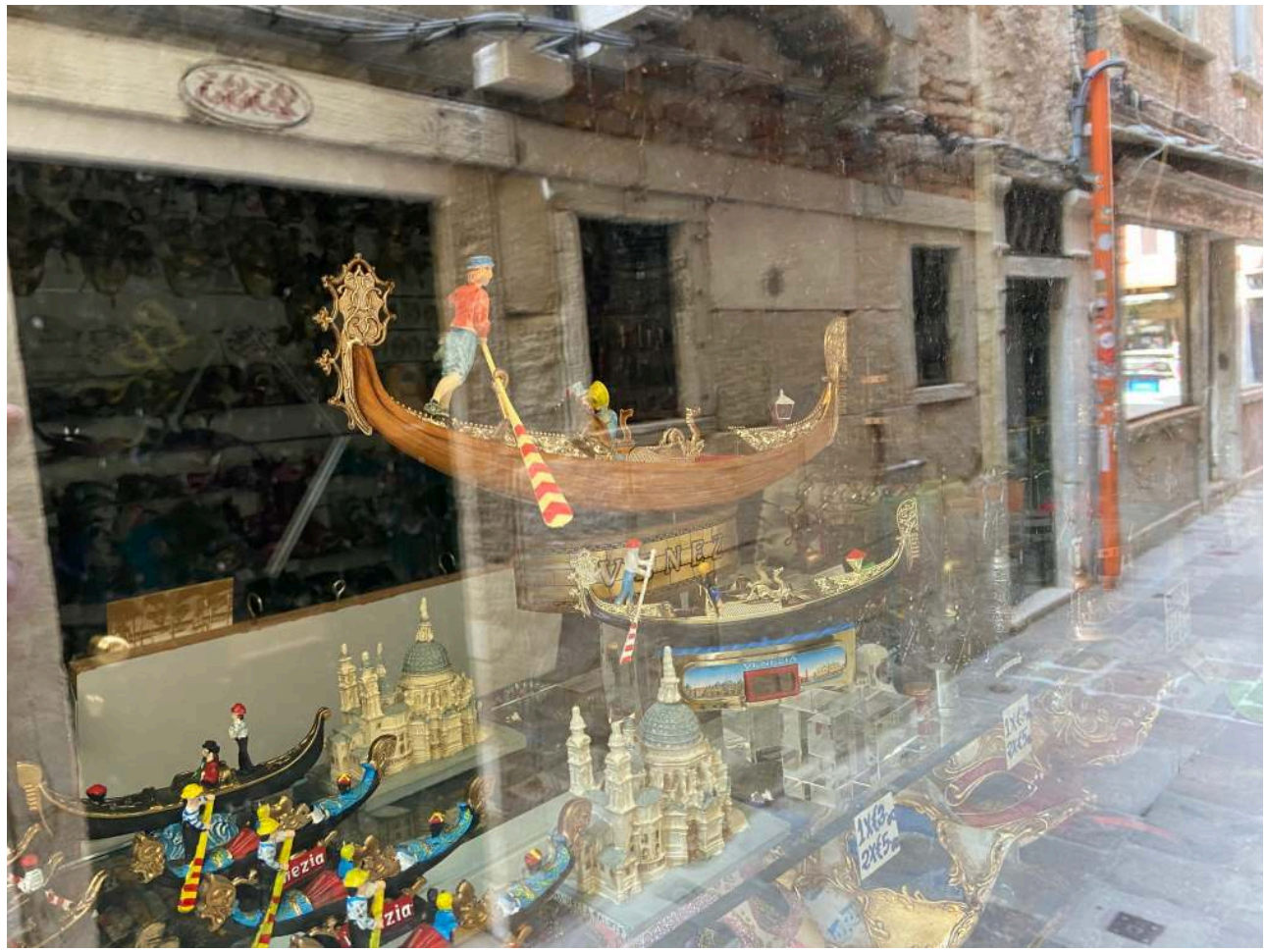

(c) Prosper Wanner, 30 avril 2020

Les « autres » visiteurs, les non-touristes, devaient, selon le protocole d'application de la taxe, être soumis à un contrôle de leur motif de visite pour savoir s'ils devaient ou 
non payer la taxe de débarquement. Certains auraient bénéficié de la vingtaine d'exemptions prévues, comme les étudiants, les résidents ou les supporters de foot. Pour tous les autres, ils auraient dû payer, dès juillet. C'est notamment le cas des clients ou partenaires des professions libérales, des amis de résidents, des stagiaires des écoles de musiques vénitiennes ou encore des clubs sportifs et d'autres activités non liées au tourisme qui, pour certaines, ont déjà annoncé qu'elles iront s'installer sur la terre ferme pour pouvoir continuer à exercer leurs activités. Les voyageurs impécunieux auraient eu comme alternative de venir à la basse saison quand les billets étaient à leur minimum, à 3 euros. Alors que la construction d'hôtels se poursuit, malgré la crise sanitaire, les "autres " voyageurs ont à leur disposition à Venise, en dehors de leurs éventuels proches, moins de 3000 lits. À titre de comparaison, fin 2019, l'observatoire citoyen indépendant du logement et de la résidence, OCIO, recensait 76273 lits touristiques sur toute la commune. En décembre 2019, j'ai organisé, avec l'association Faro Venezia, une balade patrimoniale sur l'hospitalité non touristique à Venise ${ }^{15}$. Avec l'association, nous avons identifié 24 lieux d'hospitalité non touristiques dans la ville historique, dont la moitié dédiée aux étudiants. Les autres lieux accueillent les artistes, les chercheurs, le personnel de l'hôpital, les croyants, les participants de séminaire ou les personnes en difficulté comme les femmes et mineurs isolés, les SDF et les réfugiés. La Foresteria Canal al Pianto, qui accueille le personnel hospitalier, des étudiants en médecine et les aidants, propose au total 43 lits. Elle affiche complet à l'année, sans communiquer, et refuse de nombreuses demandes.

Certains avancent que le projet de taxe de débarquement serait devenu inutile depuis que le président de la région a réussi, en 2019, à imposer la gratuité pour tous les habitants de la région et qu'une réduction a été obtenue pour les touristes qui payent déjà leur taxe de séjour ailleurs dans la région. Le risque serait in fine de créer une machinerie administrative coûteuse pour peu de cas. Des collectifs d'habitants réclament de plutôt plafonner le nombre de visiteurs, qu'ils payent ou non, ou de limiter le contrôle aux groupes de touristes arrivant en autocars, navires de croisière et bateaux-bus. D'autres villes, comme Barcelone, ont choisi de privilégier le droit à la ville. Cette logique d'optimisation du panier moyen de dépenses s'appelle en tourisme le revenu management. Il s'agit de créer des barrières tarifaires, physiques ou contractuelles, pour optimiser la rentabilité touristique. Le revenu management a été inventé par l'industrie aérienne, puis par le tourisme dans les années 1980 ; il s'agit d'une tarification dynamique basée sur l'offre et la demande. De nos jours, certains systèmes sont automatisés et vont jusqu'à tenir compte de la météo, de la hauteur des vagues et de la capacité à payer de leurs clients pour fixer les prix. Qu'importe le motif de l'hospitalité, c'est le «marché » qui fixe le prix. Cette logique a conduit les tarifs hôteliers à avoir une croissance deux fois supérieure à celle du reste du marché. Venise est devenue, par cette logique, la ville touristique la plus chère d'Italie. C'est ce qui explique en partie le succès de l'arrivée d'Airbnb qui a su, dans les villes hypertouristiques, proposer des appartement "moins cher qu'à l'hôtel » avec une cuisine, permettant d'éviter les restaurants, afin de monopoliser l'offre avant d'appliquer à son tour le revenu management et d'augmenter ses tarifs. L'intermédiation, légale ou illégale, est au cœur du système touristique, qu'il soit local ou international. Le portier d'hôtel, les plateformes numériques, les tours opérateurs, les agences de voyages locales comme internationales fonctionnent avec des commissions. Chaque intermédiaire défend sa position en limitant au maximum le contact direct entre les personnes. Bruno Latour et Michel Callon écrivaient en introduction de l'ouvrage 
Sociologie des agencements marchands que, pour passer au régime capitaliste, il suffisait de faire deux petites transformations: "traiter les proches comme de parfaits étrangers avec lesquels on sera quitte et traiter les étrangers lointains comme des intimes avec lesquels on ne sera jamais quitte ». Dans le tourisme, les voyageurs semblent in fine avoir des liens plus durables et intimes avec les intermédiaires qui gèrent leur voyage - plateformes, agences, guides - qu'avec les habitants des endroits qu'ils visitent.

\section{Apprendre à vivre « pharmacologiquement »}

Le tourisme semble s'être révélé, dans cette période hors tourisme, comme un pharmakon, c'est-à-dire "à la fois » un remède, un poison et un bouc émissaire d'une ville dans l'impasse. Pour Bernard Stiegler, «il n'y a pas de simple pharmakon » (Stiegler, 2007, p. 27). Si c'est grâce au tourisme que Venise est sortie de la crise industrielle et si c'est à cause de lui qu'elle est devenue le symbole du surtourisme, l'arrêt brutal du tourisme a rendu patente l'incurie de la ville à vivre avec le tourisme. Il ne s'agit pas de juger le tourisme, qui est tout «à la fois » remède et poison et dont l'addiction peut s'avérer parfois nécessaire, mais plutôt d'apprendre à vivre pharmacologiquement avec lui, en agissant pour que le remède soit supérieur au poison. L'incurie peut exister lorsque la société se concentre sur les seuls effets de l'addiction, sans prendre en compte ses causes. Dans le cas de Venise, les effets du surtourisme ayant disparu, ses causes sont devenues plus visibles et, in fine, elles ont permis de mieux nommer les incuries publiques comme privées, ainsi que les boucs émissaires.

27 Avec le confinement, les anti-touristes espèrent que la ville va savoir apprécier cette parenthèse sans touristes pour s'imaginer un nouvel avenir. Les pro-touristes espèrent que chacun va se rendre compte de l'importance économique du tourisme pour stimuler une reprise rapide. La question est de savoir si le confinement va contribuer à faire évoluer des positions jusque-là fortement contradictoires et disjointes vers davantage de convergence afin d'apprendre à vivre pharmacologiquement avec le tourisme. Sur les réseaux sociaux, les habitants se demandent avec quel tourisme recommencer. Dans la presse internationale et sur les réseaux sociaux, des articles racontent l'espoir d'un nouveau récit d'une Venise convertie au tourisme durable. Le nombre de touristes y serait limité et la ville privilégierait les longs séjours pour des personnes sensibles à son caractère "unique ». Dans Il Manifesto, l'écrivain vénitien Roberto Ferrucci a écrit durant ce premier confinement : la colpa è di noi veneziani (c'est de notre faute à nous, Vénitiens). Il traduit un sentiment de plus en plus partagé et énoncé. Venise serait devenue, avec le tourisme, une «slot machine » toujours gagnante. Un local ou un appartement acheté ou hérité devient rapidement un bon placement financier. De nombreux amis, qui étaient auparavant architectes, sont aujourd'hui devenus agents immobiliers et gèrent plusieurs appartements touristiques. L'hospitalité vénitienne est devenue, depuis le début du siècle, essentiellement touristique. Ses standards, ses modalités de fixation des prix et son système d'intermédiation ne s'adressent qu'au seul voyageur «touriste» au détriment des « autres voyageurs » qui visitent également une ville pour y travailler, y habiter plus ou moins temporairement, y être assistés ou y étudier (Rabbiosi et Wanner, 2020). Au-delà du cas emblématique de Venise, le tourisme est une activité d'accueil de voyageurs 
privilégiés. En France, plus du tiers de la population ne part pas en séjours touristiques, souvent faute de pouvoir se les payer. L'Organisation mondiale du tourisme a adopté une définition de la catégorie «touriste " qui reste inchangée depuis quasiment un siècle. Elle distingue, parmi les voyageurs, ceux qui viennent pour consommer sur place de ceux qui viennent travailler, étudier ou se réfugier. Pour bénéficier d'un visa touristique vers la France, il faut disposer de "ressources financières suffisantes ». La crise actuelle semble montrer, à Venise, les limites d'une régulation de l'offre d'hospitalité sur le seul critère du panier moyen de dépenses. L'exemple de Venise montre également que la définition d'une catégorie spécifique de voyageurs, aux niveaux statistique, réglementaire et fiscal - les «touristes»-, semble agir performativement sur les lieux, en invisibilisant de l'espace urbain ceux qui ne rentrent pas dans la définition, comme les travailleurs saisonniers, les étudiants, les migrants et toute une série d'autres identités mobiles (Rabbiosi et Wanner, 2020).

Photo 10. Venise, Dorsoduro, marché paysan

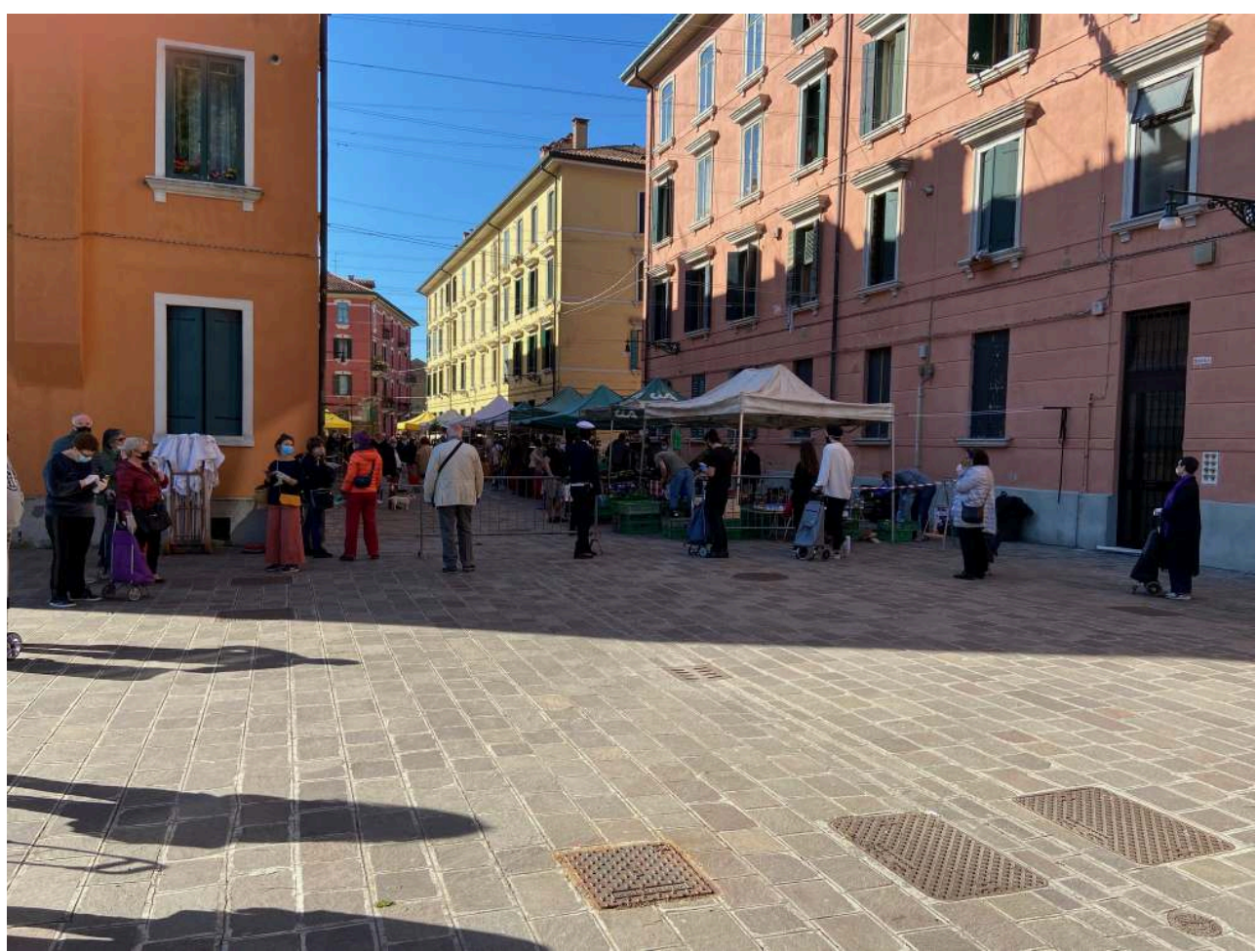

(C) Prosper Wanner, 3 mai 2020

\section{D'autres pharmaka sont-ils possibles?}

Le confinement n'a pas seulement rendu visibles des causes du surtourisme jusque-là peu prises en compte, il a aussi contribué à faire émerger d'autres imaginaires possibles. Avant la crise du Covid-19, les propositions de la ville ont porté essentiellement sur la lutte contre les effets du tourisme. La crise l'oblige à ouvrir le débat sur les causes qui l'ont amenée dans cette situation tragique, notamment l'intermédiation touristique et le libre marché foncier. Aujourd'hui, les secteurs privé comme public mesurent de facto leur dépendance à l'économie du tourisme, tout comme ils semblent redécouvrir les autres passagers. Les derniers attendus sont les 
premiers à faire tourner l'économie locale: les habitants, travailleurs, étudiants, apprentis. Cela s'accompagne d'une évolution tarifaire des offres d'hospitalité qui ne sont plus seulement calées sur la saisonnalité touristique. Les loyers d'habitation et commerciaux sont renégociés pour les mois, voire les années à venir. À Venise, la solidarité est (ré)apparue dans le secteur même du tourisme en offrant l'hospitalité au personnel soignant et aux personnes en quarantaine. Les tarifs ne sont plus fixés que par la seule loi du marché mais en vertu d'une solidarité avec les autres, allant parfois jusqu'à la gratuité. L'hospitality semble être redevenue hospitalité.

L'avenir des pratiques d'hospitalité à Venise est difficile à prévoir. Cette période d'hors tourisme a rendu visibles également d'autres récits, paysages et voyageurs. Reste à savoir si cela contribuera à faire évoluer les imaginaires des habitants et des institutions et à influencer la manière dont ils vont penser leur avenir. Jusqu'à la crise sanitaire, le récit touristique a dominé tous les autres récits possibles, à la fois économiquement et culturellement. L'exemple du futur de l'arsenal de Venise est parlant à cet égard ${ }^{16}$. En 2014, une centaine de Vénitiens, représentants d'associations, d'entreprises et de collectifs d'habitants, ont été réunis par la ville de Venise pour penser l'avenir de l'arsenal, dont la ville venait de récupérer en grande partie la propriété. Cette journée d'atelier participatif, organisée par le bureau Arsenal avec qui je collaborais, avait comme unique consigne d'imaginer ensemble ce que pourrait être un arsenal qui deviendrait un «morceau de ville sans tourisme ». À la fin de la journée, la principale idée proposée a été celle d'en faire un musée ouvert autour de la reconstruction $\mathrm{du}$ Buncintoro, navire vénitien brûlé par Napoléon. La réflexion collective avait abouti à proposer un nouveau musée, dans une ville qui en compte déjà une vingtaine, autour du récit de la Sérénissime, déjà largement raconté du Carnaval au Palais des Doges. Le bureau Arsenal s'est ainsi retrouvé face à un imaginaire collectif largement dominé par le récit économique et historique du tourisme: il semble n'y avoir pas d'alternatives économiques à la mise en tourisme d'une Venise Sérénissime. Et ce alors que la journée s'est déroulée au cœur de l'arsenal, dans un paysage à l'architecture majoritairement industrielle, où ont été construits des sous-marins, les premiers hydravions italiens et des navires en fer, comme en témoigne la grue Armstrong. Un arsenal où travaillaient encore, en 2014, 500 personnes, dans les chantiers de réparation navale, dans la recherche publique et militaire, dans les pavillons de la Biennale, dans des entreprises publiques et privées liées à la construction du système $\operatorname{MOSE}^{17}$ et dans les équipements sportifs présents dans l'enceinte de l'arsenal. Tout un ensemble d'activités peu ou pas liées à l'économie touristique. Ces lieux, récits et personnes, qui ouvrent sur d'autres imaginaires et futurs possibles pour la ville, ne semblent pas avoir été pris en compte.

Il est difficile de déterminer l'impact de ces paysages, personnages et récits, parfois anciens, qui sont (ré)apparus avec le confinement, sur les habitants et les institutions et leur capacité à penser leur avenir. Vont-ils se révéler des «flèches de futurité », pour reprendre les termes du philosophe Paul Ricoeur?

Le passé, en effet, n'est pas seulement le révolu, ce qui a eu lieu et ne peut plus être changé ; il est vivant dans la mémoire grâce aux flèches de futurité qui n'ont pas été tirées ou dont la trajectoire a été interrompue. Le futur inaccompli du passé constitue peut-être la part la plus riche d'une tradition. La délivrance de ce futur inaccompli du passé est le bénéfice majeur qu'on peut attendre du croisement des mémoires et de l'échange des récits. Ce sont principalement les événements fondateurs d'une communauté historique qu'il faudrait soumettre à cette lecture critique, de manière à libérer la charge d'espérance qu'il portait et que le cours 
ultérieur de l'histoire a trahie. Le passé est un cimetière de promesses non tenues, qu'il s'agit de ressusciter à la façon des ossements de la vallée de Josapha dans la prophétie d'Ézéchiel. (Ricoeur, 1992)

La crise du Covid-19 va-t-elle libérer des "futurs inaccomplis », qui pourraient se révéler autant d'opportunités de s'ouvrir à de nouveaux imaginaires permettant de dépasser l'impasse dans laquelle Venise semble se trouver ? Pour reprendre le concept de pharmakon de Bernard Stiegler, cet arrêt du tourisme pourrait alors être l'occasion pour la ville de Venise et ses habitants d'apprendre à vivre pharmacologiquement à partir des nouveaux imaginaires que la pandémie a (re)mis à l'ordre du jour, en ne se limitant pas à la recherche d'un tourisme plus durable, qui serait pharmacologiquement "bien dosé ", mais en s'ouvrant aux autres pharmaka que le tourisme a invisibilisé. Et, suivant l'invitation de l'écrivain Salvatore Settis (2014), de "elaborare una poetica del riuso che non si limiti alla monocoltura del turismo di massa » (élaborer une poétique de la réutilisation qui ne se limite pas à la monoculture du tourisme de masse). Cette capacité du tourisme à s'imposer à nos imaginaires n'est certainement pas propre à Venise. Elle gagnerait, de mon point de vue, à être documentée dans d'autres contextes pour contribuer à sortir de l'impasse dans laquelle semblent se trouver de nombreux lieux touristiques du fait de la forte dimension pharmacologique du tourisme, à la fois poison addictif, remède unique et bouc émissaire commode.

\section{BIBLIOGRAPHIE}

Dario BERTOCCHI, Nicola CAMATTI, Silvio GIOVE et Jan VAN DER BORG, « Venice and Overtourism: Simulating Sustainable Development Scenarios through a Tourism Carrying Capacity Model », Sustainability, vol. 12, n², p. 512, 2020 [https://doi.org/10.3390/su12020512].

Marc BOYER, Le tourisme de l'an 2000, Presses universitaires de Lyon, 1999.

Attilio BRILLI, Il viaggio in Italia: storia di una grande tradizione culturale, Ed. Il Mulino, 2008.

Michel CALLon et al., Sociologie des agencements marchands. Textes choisis, nouvelle édition, Presses des Mines, 2013.

Rodolphe CHRISTIN, Manuel de l'antitourisme, Écosociété, 2018.

Nicolò cosTA et Guido MARTINOTTI, « Sociological Theories of Tourism and Regulation Theory ", dans Lily Hoffman, Susan Feinstein et Dennis Judd (dir.), Cities and Visitors. Regulating People, Markets and City Space, Blackwell, 2003, p. 53-72.

Saskia COUSIN et Bertrand RÉAU, « L'avènement du tourisme de masse », Les Grands Dossiers des sciences humaines, $\mathrm{n}^{\circ} 22$, p. 14, 2011.

Michela DAL BORGO, At the Origin of Hospitality: Venetian Hostelry, Tavern and Lodging between the Fourteenth and Eighteenth Centuries, Hermes-Ir, 2015.

Giuseppe DE RITA, Una Città Speciale: Rapporto su Venezia, Ed. Marsilio, 1993. 
Giovanni FAVERo, Venezia dopo Venezia: economia e demografia urbana nel novecento, Laboratoire italien, 2014.

Marie GRAVARI-BARBAS et Sébastien JACQUOT, Atlas mondial du tourisme et des loisirs. Du Grand Tour aux voyages low cost, Éd. Autrement, 2018.

Marie-Françoise LANFANT, «L'appel à l'éthique et la référence universaliste dans la doctrine officielle du tourisme international », Revue Tiers Monde, t. XLV, n 178, p. 364-386, 2004 [https:// doi.org/10.3406/tiers.2004.5469].

Laetitia LEVANTIS, «Séjourner à Venise : des auberges populaires du XVIII ${ }^{\mathrm{e}}$ siècle aux luxueux hôtels de l'âge romantique », Cahiers d'études romanes, n 17, p. 317-355, 2007 [https://doiorg.ezproxy.u-paris.fr/10.4000/etudesromanes.911].

Chiara RABBIOSI et Prosper WANNER, «Dal “diritto alla città" al “diritto alla mobilità”. Spunti per una critica socio-spaziale della definizione di "turista" », Scritture Migranti, n 13, 2020, p. 129-153 [https://doi.org/10.6092/issn.2035-7141/11997].

Paul RICOEUR, «Quel éthos pour l'Europe ? », dans Peter Koslowski (dir.), Imaginer l'Europe. Le marché intérieur européen, tâche culturelle et économique, Cerf, 1992, p. 107-116.

Marco Antonio SABELLICo, De situ Venetiae urbis libri tres, 1492.

Giacomo-Maria SALERNO, «Estrattivismo contro il Comune. Venezia e l'economia turistica », ACME: An International Journal for Critical Geographies, vol. 17, n² 2, p. 480-505, 2018 [https://acmejournal.org/index.php/acme/article/view/1489].

Rosa SALZBERG, « Mobility, Cohabitation and Cultural Exchange in the Lodging Houses of Early Modern Venice », Urban History, vol. 46, n 3, p. 398-418, 2019 [https://doi.org/10.1017/ S0963926818000536].

Salvatore SETTIS, Se Venezia muore, Ed. Einaudi, 2014.

Bernard STIEGLER. «Psychopouvoir et guerre métapsychologique : la question du pharmakon », dans Le Collectif pas de 0 de conduite, Enfants turbulents : l'enfer est-il pavé de bonnes préventions?, Érès, 2008, p. 119-130.

Bernard STIEGLER, «Questions de pharmacologie générale. Il n'y a pas de simple pharmakon », Psychotropes, vol. 13, n 3-4, p. 27-54, 2007 [https://doi.org/10.3917/psyt.133.0027].

Laurent TISSOT, «Le tourisme : de l'utopie réalisée au cauchemar généralisé ? ", Entreprises et histoire, n 47, p. 5-10, 2007 [https://doi-org.ezproxy.u-paris.fr/10.3917/eh.047.0005].

UNESCO, International Campaign for the Safeguarding of Venice: Review of Results, 1966-1992, Unesco, 1992.

Jean-Didier URBAIN, L'idiot du voyage. Histoires de touristes, Éd. Payot, 1993.

Jan VAN DER BORG, Il Turismo a Venezia nel 2012, Ville de Venise, 2013.

Prosper WANNER, «Venise, la taxe de débarquement fait des vagues », Espaces Tourisme, $\mathrm{n}^{\circ} 351$, 2019.112-121, p.

Andrea ZANNINI, Il turismo a Venezia dal secondo dopoguerra ad oggi, Laboratoire italien, 2014.

\section{NOTES}

1. Données ville de Venise, Annuaire du tourisme et données mouvement démographique. 
2. Traduction de l'auteur : « from the socio-demographic point of view, there is hypertourism every time that the city users or vacationer visitors exceed the number of residents and therefore the city is defined as a place with a basic tourist vocation (...) similar to a theme park ".

3. UNESCO, International Campaign for the Safeguarding of Venice: Review of Results, 1966-1992, Unesco, 1992.

4. Région Vénétie, rapport statistique 2019, chapitre 6 (en italien): http:// statistica.regione.veneto.it/Pubblicazioni/RapportoStatistico2019/capitolo-6.html

5. Annuaire du tourisme, ville de Venise (en italien): https://www.comune.venezia.it/it/ content/studi

6. Quotidien AffarItaliani.it, juillet 2008: https://www.affaritaliani.it/cronache/ veneziacortinanoelemosina250708.html

7. En 2017, selon la responsable de l'inspection du travail de Venise, le travail au noir était en augmentation à Venise avec 596 cas constatés, le secteur de la restauration et du tourisme étant celui qui comptait le plus grand nombre d'irrégularités. Quotidien Venezia Today, 18 juillet 2018 : https://www.veneziatoday.it/economia/dati-lavoro-nero-provincia-venezia-18-luglio-2018.html

8. https://www.nationalgeographic.fr/environnement/2020/03/coronavirus-attention-aux-fakenews-sur-le-retour-de-la-vie-sauvage

9. Site de l'association Dingo, créée en 1969 : https://www.dingovenezia.it/

10. Accord entre la ville de Venise et des sociétés de taxi adopté le 18 mai 2020 : https:// live.comune.venezia.it/sites/live.comune.venezia.it/files/articoli/allegati/

city_taxi-2_70x100_16_5.pdf

11. Article d'Il Gazzettino, 6 novembre 2017: https://www.ilgazzettino.it/nordest/venezia/ turista_scrive_al_sindaco_pranzo_di_pesce_venezia-3349752.html

12. Article Nuova Venezia, 27 septembre 2019 : https://nuovavenezia.gelocal.it/venezia/cronaca/ 2019/09/27/news/accordi-tra-vetrerie-e-agenzie-turistiche-cinesi-trovato-il-nero-1.37524242

13. Article Corriere del Veneto, 12 mai 2020 : https://corrieredelveneto.corriere.it/venezia-mestre/ cronaca/20_maggio_12/venezia-cipriani-non-riapre-lunedi-condizioni-dell-inail-

demenziali-0b7544ae-9475-11ea-99bb-52457e92a1ec.shtml

14. Définition de phamarkon sur le site d'Ars Industrialis, l'association internationale pour une politique industrielle des technologies de l'esprit, créée autour des travaux de recherche de Bernard Stiegler : http://arsindustrialis.org/pharmakon

15. Article de l'association Faro Venezia sur la balade patrimoniale autour de l'hospitalité non touristique à Venise (en italien) : https://farovenezia.org/2019/12/21/passeggiata-patrimonialesullospitalita-a-venezia/

16. Article sur le processus à l'arsenal sur le site de l'association Faro Venezia (en italien) : https://farovenezia.org/2018/02/10/larsenale-18-mesi-di-percorso-faro/

17. Le système MOSE, MOdulo Sperimentale Elettromeccanico (module expérimental électromécanique), est le système de fermeture de la lagune de Venise en cas de grande marée.

\section{RÉSUMÉS}

Venise a été l'une des premières villes en Europe à être confinée. L'article propose d'observer ce moment unique de "hors tourisme " sous l'angle de la pharmacologie. La notion de pharmakon est empruntée au philosophe Bernard Stiegler comme outil d'analyse du tourisme à partir de son 
ambivalence, à la fois remède, poison et bouc émissaire. L'observation in situ des cent jours du premier confinement porte sur des lieux, des personnes et des récits qui sont (re)devenus visibles : la nature, les mendicanti (mendiants), les pendolari (navetteurs) et l'espace public. Les effets du surtourisme ayant disparu, ce hors tourisme va rendre manifeste des causes de la forte dépendance de Venise au tourisme jusque-là peu discutées, comme le marché foncier, les discriminations tarifaires et l'intermédiation touristique. Ces observations interrogent sous un nouvel angle le récit d'un surtourisme dont les mordi fuggi (ceux qui mordent et s'enfuient) seraient la principale cause et que la ville souhaite limiter à travers l'instauration d'une taxe d'entrée à la ville.

Venice was one of the first cities in Europe to be in lockdown. The article proposes to observe this unique moment "out of tourism" from the angle of pharmacology. The concept of pharmakon is borrowed from the philosopher Bernard Stiegler as a tool for analysing tourism from its ambivalence, both remedy, poison and scapegoat. The in-situ observation of the one hundred days of the first lockdown relates to places, people and stories that have become visible: nature, mendicanti (beggars), pendolari (commuters) and public space. As the effects of over-tourism have disappeared, this moment out of tourism has made manifest the causes of Venice's heavy dependence on tourism, until then little discussed, such as the property market, tariff discrimination and tourism intermediation. These observations offer a different perspective on an over-tourism whose mordi fuggi (who bite and flee) would be the main cause and which the city wishes to limit through the introduction of a city entrance tax.

\section{INDEX}

Keywords : pharmakon, overtourism, Venice, lockdown, Covid-19

Mots-clés : pharmakon, surtourisme, Venise, confinement, Covid-19

\section{AUTEUR}

\section{PROSPER WANNER}

Anthropologue

Doctorant CIFRE au sein de la SCIC Les oiseaux de passage, laboratoire CESSMA (université de Paris)

pwanner[at]lesoiseauxdepassage.coop 